\title{
Proof of the Bogoliubov-Parasiuk Theorem on Renormalization
}

\author{
KLAUS HePP*** \\ The Institute for Advanced Study, Princeton, New Jersey \\ Received February 7, 1966
}

\begin{abstract}
A new proof is given that the subtraction rules of Bogoliubov and Parasiuk lead to well-defined renormalized Green's distributions. A collection of the counter-terms in "trees" removes the difficulties with overlapping divergences and allows fairly simple estimates and closed expressions for renormalized Feynman integrals. The renormalization procedure, which also applies to conventionally nonrenormalizable theories, is illustrated in the $\varphi^{4}$-theory.
\end{abstract}

\section{Introduction}

Renormalization in Lagrangian quantum field theory is in the interpretation of Bogoliubov and Parasiuk [1], [2] the extension of certain linear functionals, defined on a subspace of $\mathscr{S}\left(R^{4 n}\right)$, to tempered distributions in $\mathscr{S}^{\prime}\left(R^{4 n}\right)$ [3]. For instance, the Gell-Mann Low perturbation expansion of the truncated time-ordered distributions has the form

$$
\begin{aligned}
& \left\langle T \varphi_{1}\left(x_{1}\right) \ldots \varphi_{m}\left(x_{m}\right)\right\rangle^{T}=\sum_{n=m}^{\infty} \frac{(-i)^{n-m}}{(n-m) !} \int d x_{m+1} \ldots d x_{n} \times \\
\times & \left\langle T \varphi_{1}^{\mathrm{I}}\left(x_{1}\right) \ldots \varphi_{m}^{\mathrm{I}}\left(x_{m}\right) \mathscr{H}^{\mathrm{I}}\left(x_{m+1}\right) \ldots \mathscr{H}^{\mathrm{I}}\left(x_{n}\right)\right\rangle^{T} .
\end{aligned}
$$

Here the truncated vacuum expectation values $\left\langle\varphi_{1}^{\mathrm{I}}\left(x_{1}\right) \ldots \mathscr{H}^{\mathrm{I}}\left(x_{n}\right)\right\rangle^{T}$ are well-defined for $\mathscr{H}^{\mathrm{I}}(x)$, which are WICK polynomials of the free fields $\varphi_{i}^{\mathrm{I}}(x)$. On the other hand the straightforward construction of $\left\langle T \varphi_{1}^{\mathrm{I}}\left(x_{1}\right) \ldots \mathscr{H}^{\mathrm{I}}\left(x_{n}\right)\right\rangle^{T}$ by WICK's theorem [4] leads to a product of distributions

$$
\prod_{l \in \mathscr{L}} \Delta_{l}^{F}\left(x_{i_{l}}-x_{f_{l}}\right)
$$

Formula (1.2) is in general not meaningful as one sees from the definition of $\Delta_{l}^{F}$ in $p$-space

$$
\widetilde{\triangle}_{l}^{F}(p)=\lim _{\varepsilon \downarrow 0} i P_{\imath}(p)\left(p^{2}-m_{l}^{2}+i \varepsilon\right)^{-1},
$$

where $P_{l}(p)$ is a polynomial and where $m_{l}>0$ is always assumed. Then the convolutions in $p$-space corresponding to (1.2) can lead to "ultraviolet divergences"'.

Nevertheless the product (1.2) taken with regularized [5] propagators is a good starting point for the definition of $\left\langle T \varphi_{1}^{\mathrm{I}}\left(x_{1}\right) \ldots \mathscr{H}^{\mathrm{I}}\left(x_{n}\right)\right\rangle^{T}$.

\footnotetext{
* Research supported by the National Science Foundation.

** Present address: ETH, Zürich, Switzerland.
} 
The choice of regulators will turn out to be rather arbitrary, but it is desirable to maintain Lorentz covariance. Using

$$
\widetilde{J} f(p)=\lim _{\varepsilon \downarrow 0} P_{l}(p) \int_{0}^{\infty} d \alpha \exp \left[i \alpha\left(p^{2}-m_{l}^{2}+i \varepsilon\right)\right]
$$

we define for $\varepsilon>0, r>0$

$$
\widetilde{U}_{l}^{r, \varepsilon}(p)=P_{l}(p) \int_{r}^{\infty} d \alpha \exp \left[i \alpha\left(p^{2}-m_{l}^{2}+i \varepsilon\right)\right] .
$$

$\chi_{r, 8}$ belongs to $\mathcal{O}_{M}\left(R^{4}\right) \cap \mathcal{O}_{C}^{\prime}\left(R^{4}\right)$ ([3], vol. 2, p. 101) and Fourier transforms and convolutions of several $\widetilde{U}_{l}^{r, 8}$ can be obtained (in the sense of distribution theory) by interchange with the $\alpha$-integration. Evidently $\widetilde{J}_{l}(p)-\lim _{\varepsilon \downarrow 0} \widetilde{J}_{l}, \varepsilon(p)$ converges to zero in $\mathcal{O}_{M}\left(R^{4}\right)$ for $r \downarrow 0$. One has

$$
\Delta_{l}^{r, 8}(x)=-\frac{i}{4} P_{l}\left(i \frac{\partial}{\partial x}\right) \cdot \int_{r}^{\infty} \frac{d \alpha}{\alpha^{2}} \exp \left[-i \alpha\left(m_{l}^{2}-i \varepsilon\right)-i \frac{x^{2}}{4 \alpha}\right]
$$

in $\mathcal{O}_{M}\left(R^{4}\right) \cap \mathcal{O}_{C}^{\prime}\left(R^{4}\right)$ and thus the product $\prod_{l \in \mathscr{L}} \Delta_{l}^{\tau, \varepsilon}\left(x_{i_{l}}-x_{f_{l}}\right)$ is welldefined. Furthermore it can be shown ([6]; see sec. 4) that

$$
\lim _{\varepsilon \downarrow 0} \lim _{r \downarrow 0} \prod_{l \in \mathscr{L}} \Delta_{l}^{r, \varepsilon}\left(x_{i_{l}}-x_{f_{l}}\right)
$$

is a continuous linear functional on the subspace $\mathscr{S}_{N}\left(R^{4 n}\right)$ of those test functions $\varphi \in \mathscr{S}\left(R^{4 n}\right)$, which vanish of sufficiently high order $N$ whenever two arguments $x_{i}, x_{j}, 1 \leqq i<j \leqq n$, coincide.

The renormalization theory of Dyson [7] is in this framework a constructive form of the Hahn-Banach theorem: one subtracts from $\Pi \Delta_{l},{ }^{,}\left(x_{i_{l}}-x_{f_{l}}\right)$ counter terms which vanish on $\mathscr{S}_{N}\left(R^{4 n}\right)$, such that the remainder has a limit in $\mathscr{S}^{\prime}\left(R^{4 n}\right)$ for $r \downarrow 0$ and $\varepsilon \downarrow 0$. The fact that these subtractions can be implemented by formal counter terms in $\mathscr{H}^{\mathrm{I}}(x)$ is an inherently beautiful feature of Lagrangian quantum field theory.

Feynman graphs efficiently organize the combinatorics of the counter terms. We map the $n$ arguments $x_{1}, \ldots x_{n}$ of $\prod_{v=1}^{L} \Delta_{l_{\nu}}^{r, 8}\left(x_{i_{v}}-x_{f_{v}}\right)$ onto $n$ points $V_{1}, \ldots V_{n}$ in a plane, called vertices, and each propagator $\Delta_{i v}^{r, 8}\left(x_{i_{v}}-x_{i_{v}}\right)$ on an oriented line $l_{v}$ from $V_{i_{v}}$ to $V_{t_{v}}$. This gives (up to topological equivalence) the graph $G\left(V_{1}, \ldots V_{n}, \mathscr{L}\right)$ for $\Pi \Delta_{l}^{\varepsilon, r}$, where $\mathscr{L}=\left\{l_{1}, \ldots l_{L}\right\}$.

Def.: A subset $U=\left\{V_{1}^{\prime}, \ldots V_{m}^{\prime}\right\} \subset\left\{V_{1}, \ldots V_{n}\right\}$ is called a generalized vertex of $G\left(V_{1}, \ldots V_{n}, \mathscr{L}\right)$.

Def.: Let $U_{i}=\left\{V_{i 1}, \ldots V_{i r(i)}\right\}, 1 \leqq i \leqq m$, be pairwise disjoint generalized vertices of $G\left(V_{1}, \ldots V_{n}, \mathscr{L}\right)$ and $\mathscr{M} \subset \mathscr{L}$. Then the graph $G\left(U_{1}, \ldots U_{m}, \mathscr{M}\right)$ is obtained by representing the sets $U_{1}, \ldots U_{m}$ by $m$ points in a plane (again denoted by $U_{i}$ ) and by connecting them by 
those $l \in \mathscr{M}$, which run between different $U_{i}$ (i.e. between $V_{i_{l}} \in U_{i}$, $\left.V_{f_{l}} \in U_{f}, U_{i} \neq U_{f}\right)$. Sets $\left\{V_{i}\right\}$ are also denoted by $V_{i}$.

Example: Let $\mathscr{L}=\left\{l_{1}, \ldots l_{6}\right\}$ and $G\left(V_{1}, \ldots V_{4}, \mathscr{L}\right)$ be as in Fig. 1 . If $U_{1}=\left\{V_{1}, V_{2}\right\}$ and $\mathscr{M}=\left\{l_{1}, l_{4}, l_{5}, l_{6}\right\}$, then $G\left(U_{1}, V_{3}, \mathscr{M}\right)$ becomes Fig. 1.
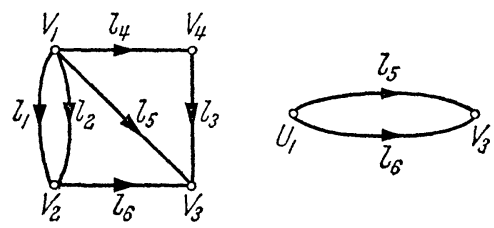

Fig. 1. Graph and generalized subgraph

Def.: $G\left(U_{1}, \ldots U_{m}, \mathscr{M}\right)$ is connected, if $\left\{U_{1}, \ldots U_{m}\right\}$ is connected by the lines in $\mathscr{M} . G\left(U_{1}, \ldots U_{m}, \mathscr{M}\right)$ is 1-particle irreducible (IPI), if for all $l \in \mathscr{M}$ and $\mathscr{M} / l=\mathscr{M}-\{l\}, G\left(U_{1}, \ldots U_{m}, \mathscr{M} / l\right\}$ is connected. Otherwise $G\left(U_{1}, \ldots U_{m}, \mathscr{M}\right)$ is called 1-particle reducible (IPR).

Def.: The superficial divergence $v\left(V_{1}^{\prime}, \ldots V_{m}^{\prime}\right)$ of $G\left(V_{1}^{\prime}, \ldots V_{m}^{\prime}, \mathscr{L}\right)$, $\left\{V_{1}^{\prime}, \ldots V_{m}^{\prime}\right\} \subset\left\{V_{1}, \ldots V_{n}\right\}$, is defined by

$$
v\left(V_{1}^{\prime}, \ldots V_{m}^{\prime}\right)=\sum_{\text {conn }}\left(r_{l}+2\right)+4(m-1)
$$

where $\sum_{\text {conn }}$ extends over all $l \in \mathscr{L}$, which connect two vertices from $\left\{V_{1}^{\prime}, \ldots V_{m}^{\prime}\right\}$ and $r_{l}$ is the degree of the polynomial $P_{l}$ in (1.3). In the above example one has for a scalar theory $\left(r_{l}=0\right)$ :

$$
v\left(V_{1}, V_{2}\right)=0, v\left(V_{1}, V_{2}, V_{3}\right)=0, v\left(V_{1}, V_{2}, V_{3}, V_{4}\right)=0 .
$$

To each generalized vertex $\left\{V_{1}^{\prime}, \ldots V_{m}^{\prime}\right\}$ we define a vertex part $\mathscr{X}_{\mathscr{L}}^{r, \varepsilon}\left(V_{1}^{\prime}, \ldots V_{m}^{\prime}\right)$ as the following distribution with support in $\left\{x_{1}^{\prime}=\cdots=x_{m}^{\prime}\right\}$ :

Def.: Let $\left\{V_{1}^{\prime}, \ldots V_{m}^{\prime}\right\} \subset\left\{V_{1}, \ldots V_{n}\right\}$. Then

$$
\begin{gathered}
\mathscr{X}_{\mathscr{L}}^{r, \varepsilon}\left(V_{1}^{\prime}, \ldots V_{m}^{\prime}\right)= \begin{cases}1 & , \text { if } m=1 \\
0 \quad & \text { for } \operatorname{IPR} G\left(V_{1}^{\prime}, \ldots V_{m}^{\prime}, \mathscr{L}\right) \\
-M \overline{\mathscr{R}}_{\mathscr{L}}^{r, \varepsilon}\left(V_{1}^{\prime}, \ldots V_{m}^{\prime}\right), & \text { otherwise },\end{cases} \\
\overline{\mathscr{R}}_{\mathscr{L}}^{r, \varepsilon}\left(V_{1}^{\prime}, \ldots V_{m}^{\prime}\right)=\sum_{P}^{\prime} \prod_{j=1}^{k(P)} \mathscr{X}_{\mathscr{L}}^{r, \varepsilon}\left(V_{j 1}^{P}, \ldots V_{j r(j)}^{P}\right) \prod_{\text {conn }} \Delta^{r, \varepsilon} .
\end{gathered}
$$

Here $\sum_{P}^{\prime}$ extends over all partitions $\left\{\left\{V_{j 1}^{P}, \ldots V_{j r(j)}^{P}\right\}, 1 \leqq j \leqq k(P)\right\}$, of $\left\{V_{1}^{\prime}, \ldots V_{m}^{\prime}\right\}$ into $1<k(P) \leqq m$ sets and $\Pi_{\text {conn }}$ is taken over all $l \in \mathscr{L}$ which connect different sets of the partition. The operation $M$ maps $\overline{\mathscr{R}}_{\mathscr{L}}^{r, \varepsilon}\left(V_{1}^{\prime}, \ldots V_{m}^{\prime}\right)$, being in $p$-space of the form $\delta\left(p_{1}^{\prime}+\cdots+p_{m}^{\prime}\right) \times$ $\times F\left(p_{1}^{\prime}, \ldots p_{m}^{\prime}\right)$ with $F \in \mathcal{O}_{M}\left(R^{4 m}\right)$, into $\delta\left(p_{1}^{\prime}+\cdots+p_{m}^{\prime}\right) T\left(p_{1}^{\prime}, \ldots p_{m}^{\prime}\right)$, where the polynomial $T$ is the Taylor series of $F$ around $p_{1}^{\prime}=\cdots p_{m}^{\prime}=0$

Commun. math. Phys., Vol. 2 
up to the order $v\left(V_{1}^{\prime}, \ldots V_{m}^{\prime}\right), T=0$ for $v<0$. One verifies by induction that for $\varepsilon>0, r>0$ the above definition makes sense. Finally we set

$$
\mathscr{R}_{\mathscr{L}}^{r, \varepsilon}\left(V_{1}^{\prime}, \ldots V_{m}^{\prime}\right)=\overline{\mathscr{R}}_{\mathscr{L}}^{r, \varepsilon}\left(V_{1}^{\prime}, \ldots V_{m}^{\prime}\right)+\mathscr{X}_{\mathscr{L}}^{r, \varepsilon}\left(V_{1}^{\prime}, \ldots V_{m}^{\prime}\right) \text {. }
$$

Example: If we denote graphically each $(-M)$-operation by a dotted encircling of the corresponding subgraph, then we obtain in a typical case Fig. 2. We have only listed the non-vanishing terms. One sees the
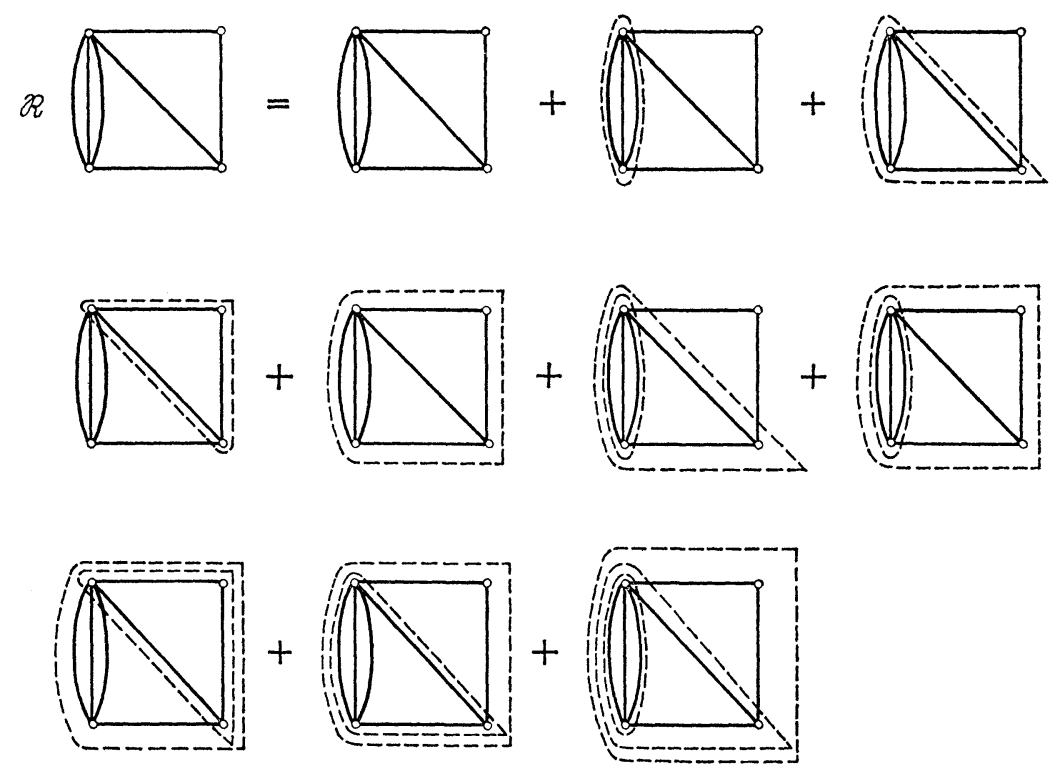

Fig. 2. Counterterms

emergence of subtraction rules, which are similar but not identical with those proposed by SaLam [8].

The main theorem of Bogoliubov and Parasiuk is that for an arbitrary product of Feynman propagators (1.2) $\lim _{\varepsilon \downarrow 0} \lim _{r \downarrow 0} \mathscr{R}_{\mathscr{L}}^{r, \varepsilon}\left(V_{1}, \ldots V_{n}\right)$ exists as a tempered distribution and defines a Lorentz covariant continuation of (1.2) which can be implemented by (formal) counter terms in $\mathscr{H}^{\mathrm{I}}(x)$.

Unfortunately the papers of Bogoliubov and Parasiuk [1], [2] come close to not satisfying SaLAM's criterion: it is hard to find two theoreticians whose understanding of the essential steps of the proof is isomorphic. This is particularly regrettable, since the very ingenious and elaborate treatment of the authors is the most general discussion of renormalization in Lagrangian quantum field theory. Our aim is to give a new and possibly clearer account of the fundamental operations and to 
circumvent a number of errors in the original proof of the main theorem. We shall give a new treatment of the combinatorial structure of the $\mathscr{R}$-operation (Lemma 2.4), which deals efficiently with the overlapping divergences and leads to a direct majorization of the counter terms in partial sums of tree structure (Lemma 3.2). Each tree gives a renormalized Feynman integral in the limit $r \downarrow 0$, which has the usual analyticity properties and is a tempered distribution for $\varepsilon \downarrow 0$. In an example we shall discuss the renormalized perturbation series for the $\varphi^{4}$-theory [9].

\section{Tree structure of the $\mathscr{R}$-0peration}

In momentum space each term in $\mathscr{R}_{\mathscr{L}}^{r, \varepsilon}\left(V_{1}, \ldots V_{n}\right)$ has the following structure

$\delta\left(\sum p_{i}\right) \int_{r}^{\infty} \ldots \int_{r}^{\infty} \prod_{l=1}^{L} d \alpha_{l} R(\alpha, p) \exp \left[i \sum_{1}^{n} A_{i j} p_{i} p_{j}-i \sum_{1}^{L} \alpha_{l}\left(m_{l}^{2}-i \varepsilon\right)\right]$

The $A_{i j}=A_{i j}(\alpha)$ are rational and homogeneous of degree +1 and $R(\alpha, p)$ rational and in general not locally $\alpha$-integrable for $\alpha \downarrow 0$. Thus the "ultraviolet divergences" become manifest and the counter terms are introduced to enforce local integrability when $r \downarrow 0$. The greatest difficulty in proving the existence of $\lim _{\varepsilon \downarrow 0} \lim _{r \downarrow 0} \mathscr{R}_{\mathscr{L}}^{r, \varepsilon}\left(V_{1}, \ldots V_{n}\right)$ arises from "overlapping divergences", i.e. from the necessity of subtractions for generalized vertices $U_{i}=\left\{V_{i 1}, \ldots V_{i k}\right\}, \quad U_{j}=\left\{V_{j 1}, \ldots V_{j l}\right\}$ with $U_{i} \cap U_{j} \neq \emptyset$ and neither $U_{i} \supset U_{j}$ nor $U_{j} \supset U_{i}$. Although each term in $\mathscr{R}_{\mathscr{L}}^{r, \delta}\left(V_{1}, \ldots V_{n}\right)$ diverges in general for $r \downarrow 0$, one easily sees in simple examples that certain partial sums of counter terms converge individually in regions of the type $\alpha_{l_{1}} \geqq \cdots \geqq \alpha_{l_{L}} \geqq 0$.

This leads to the following combinatorial problem: Given an ordering $l_{1}>\cdots>l_{L}$ of the lines $\mathscr{L}$. Can one decompose $\mathscr{R}_{\mathscr{L}}^{r, \varepsilon}\left(V_{1}, \ldots V_{n}\right)$ into partial sums involving only non-overlapping subtractions, while keeping definite order relations between the lines $\mathscr{L}$ such that "the right subtractions arise at the right places" ? The answer is affirmative, and the problem of keeping track of all counter terms has found a surprisingly simple solution by Lemma 2.2 due to Bogoliubov and Parasiuk [1], [2]. In this section we shall not denote explicitly the $r>0$ and $\varepsilon>0$ dependence (by writing e.g. $\Delta_{l}$ for $\Delta_{l}^{r, \varepsilon}$ ).

We first introduce a generalized $\mathscr{R}$-operation for a set $U_{1}, \ldots U_{s}$ of pairwise disjoint generalized vertices $U_{i}=\left\{V_{i 1}, \ldots V_{i r(i)}\right\}$ with vertex parts $\mathscr{X}\left(U_{i}\right)$ (i.e. distributions with support in $\left.\left\{x_{i 1}=\cdots=x_{i r(i)}\right\}\right)$ and for a subset $\mathscr{M} \subset \mathscr{L}$. For any union $U_{1}^{\prime} \cup \cdots \cup U_{t}^{\prime}, U_{i}^{\prime} \in\left\{U_{1}, \ldots U_{s}\right\}$, we 
define a vertex part as in $(1.8,9,10)$ by

$$
\begin{aligned}
\mathscr{X}_{\mathscr{M}}\left(U_{1}^{\prime}, \ldots U_{t}^{\prime}\right) & =\left\{\begin{array}{l}
\mathscr{X}\left(U_{1}^{\prime}\right) \text { if } t=1 \\
0 \text { if } G\left(U_{1}^{\prime}, \ldots U_{t}^{\prime}, \mathscr{M}\right) \text { is IPR } \\
-M \overline{\mathscr{R}}_{\mathscr{M}}\left(U_{1}^{\prime}, \ldots U_{t}^{\prime}\right) \text { otherwise },
\end{array}\right. \\
\overline{\mathscr{R}}_{\mathscr{M}}\left(U_{1}^{\prime}, \ldots U_{t}^{\prime}\right) & =\sum_{P}^{\prime} \prod_{j=1}^{k(P)} \mathscr{X}_{\mathscr{M}}\left(U_{j 1}^{P}, \ldots U_{j r(j)}^{P}\right) \prod_{\text {conn }} \Delta_{l} .
\end{aligned}
$$

$\sum_{P}^{\prime}$ runs over all partitions $P$ of $\left\{U_{1}^{\prime}, \ldots U_{t}^{\prime}\right\}$ into $1<k(P) \leqq t$ sets and $\Pi$ over all lines $l \in \mathscr{L}$, for which $V_{i_{l}}$ and $V_{f_{l}}$ lie in different sets of the conn partition. $M$ is defined in $p$-space as Taylor expansion of the coefficient of $\delta\left(\sum_{i, j} p_{i j}\right)$ up to the order $v\left(V_{11}, \ldots V_{t k(t)}\right)$ around the origin. Finally

$$
\mathscr{R}_{\mathscr{M}}\left(U_{1}^{\prime}, \ldots U_{t}^{\prime}\right)=\overline{\mathscr{R}}_{\mathscr{M}}\left(U_{1}^{\prime}, \ldots U_{t}^{\prime}\right)+\mathscr{X}_{\mathscr{M}}\left(U_{1}^{\prime}, \ldots U_{t}^{\prime}\right) .
$$

It is important to observe that $\mathscr{M}$ only accounts for IPI in (2.2), while $\Pi$ extends over all connecting lines $l \in \mathscr{L}$ and $\mathscr{L}$ determines conn

$\nu\left(V_{11}, \ldots V_{t k(t)}\right)$ by (1.7). Thus by varying $\mathscr{M}$ one changes the number of counter terms in (2.4) without affecting the analytical form of the remaining terms.

Lemma 2.1. If $G\left(U_{1}, \ldots U_{s}, \mathscr{M}\right)$ is IPR, then there exists a unique partition of $\left\{U_{1}, \ldots U_{s}\right\}$ into $\left\{U_{01}\right\}, \ldots\left\{U_{0 s(0)}\right\},\left\{U_{11}, \ldots U_{1 s(1)}\right\}, \ldots$ $\ldots\left\{U_{r 1}, \ldots U_{r s(r)}\right\}, s(1), \ldots s(r)>1$, such that $G\left(U_{i 1}, \ldots U_{i s(i)}, \mathscr{M}\right)$ are IPI and

$$
\begin{aligned}
\mathscr{R}_{\mathscr{M}}\left(U_{1}, \ldots U_{s}\right) & =\overline{\mathscr{R}}_{\mathscr{M}}\left(U_{1}, \ldots U_{s}\right) \\
= & \prod_{i=1}^{s(0)} \mathscr{X}\left(U_{0 i}\right) \prod_{j=1}^{r} \mathscr{R}_{\mathscr{M}}\left(U_{j 1}, \ldots U_{j s(j)}\right) \prod_{\text {conn }} \Delta_{l},
\end{aligned}
$$

where $\underset{\text { conn }}{\Pi}$ extends over all $l \in \mathscr{L}$ connecting generalized vertices from different sets of the partition.

Proof: One obtains trivially the decomposition of $G\left(U_{1}, \ldots U_{s}, \mathscr{M}\right)$ into IPI components by looking at the corresponding graph. These components are some generalized vertices $U_{0 i}$ and some IPI subgraphs $G\left(U_{j 1}, \ldots U_{j s(j)}, \mathscr{M}\right)$. Since the definition of $\mathscr{R}_{\mathscr{M}}\left(U_{1}, \ldots U_{s}\right)$ excludes all partitions of $\left\{U_{1}, \ldots U_{s}\right\}$, which are not finer than $\left\{U_{01}\right\}, \ldots$ $\ldots\left\{U_{r 1}, \ldots U_{r s(r)}\right\}$ (because otherwise there would appear at least one IPR subgraph) one obtains (2.5).

Since the connecting lines between the IPI components of any $G\left(V_{1}, \ldots V_{n}, \mathscr{L}\right)$ do not form closed loops, we can in the sequel restrict ourselves to the renormalization of IPI graphs. The following lemma shows how to regroup the counter terms in $\mathscr{X}_{\mathscr{M}}\left(U_{1}, \ldots U_{m}\right)$, if $\mathscr{M}$ is replaced by $\mathscr{M} / l$ : 
Lemma 2.2: Given $\mathscr{M} \subset \mathscr{L}$ and a disjoint set of generalized vertices $U_{1}, \ldots U_{m}$ with vertex parts $\mathscr{X}\left(U_{i}\right)$. If $l \in \mathscr{M}$ connects different $U_{i}$, then $\mathscr{X}_{\mathscr{M}}\left(U_{1}, \ldots U_{m}\right)$

$=\mathscr{X}_{\mathscr{M} l l}\left(U_{1}, \ldots U_{m}\right)+\sum_{j} \mathscr{X}_{\mathscr{M} / l}\left(U_{j 1} \cup \cdots \cup U_{j r(j)}, U_{j(r(j)+1)}, \ldots U_{j m}\right)$

Here $\sum_{j}$ extends over all IPI $G\left(U_{j 1}, \ldots U_{j r(j)}, \mathscr{M}\right), 1<r(j) \leqq m$, which become IPR without $l . \mathscr{X}_{\mathscr{M} / l}\left(U_{j 1} \cup \cdots \cup U_{j r(j)}, \ldots U_{j m}\right)$ is defined by (2.2) starting from $\mathscr{X}\left(U_{j k}\right), k>r(j)$, and from new vertex parts for $U_{j 1} \cup \cdots \cup U_{j r(j)}:$

$$
\mathscr{X}_{\mathscr{M} / l}\left(U_{j 1} \cup \cdots \cup U_{j r(j)}\right)=-M \overline{\mathscr{R}}_{\mathscr{M} / l}\left(U_{j 1}, \ldots U_{j r(j)}\right) .
$$

Proof $^{1}$ by induction: The lemma is true for $m=1$. We assume that Lemma 2.2 holds for all proper subsets $\left\{U_{1}^{\prime}, \ldots U_{k}^{\prime}\right\}$ of $\left\{U_{1}, \ldots U_{m}\right\}$, where $k<m$ and $l \in \mathscr{M}<\mathscr{L}$ is fixed.

If $G\left(U_{1}, \ldots U_{m}, \mathscr{M}\right)$ is IPR, then also $G\left(U_{1}, \ldots U_{m}, \mathscr{M} / l\right)$. Since any IPI $G\left(U_{j 1}, \ldots U_{j r(j)}, \mathscr{M}\right)$ must lie in a IPI component of $G\left(U_{1}, \ldots U_{m}, \mathscr{M}\right)$, all the graphs $G\left(U_{j 1} \cup \cdots \cup U_{j r(j)}, \ldots U_{j m}, \mathscr{M} / l\right)$ are IPR. Therefore both sides of (2.6) vanish.

If $G\left(U_{1}, \ldots U_{m}, \mathscr{M}\right)$ is IPI and $m>1$, then we use (2.2) and (2.3). For each of the $\mathscr{X}_{\mathscr{M}}$ on the right hand side of (2.3) the induction hypothesis applies. If $l$ does not connect two vertices from $U_{j 1}^{P}, \ldots U_{j r(j)}^{P}$, then obviously $\mathscr{X}_{\mathscr{M}}\left(U_{j 1}^{P}, \ldots U_{j r(j)}^{P}\right)=\mathscr{X}_{\mathscr{M} l l}\left(U_{j 1}^{P}, \ldots U_{j r(j)}^{P}\right)$. The other alternative occurs at most once in every product in (2.3), say for $j=1$. By the induction hypothesis (2.3) becomes

$$
\begin{aligned}
\mathscr{X}_{\mathscr{M}}\left(U_{1}, \ldots U_{m}\right)=-M \overline{\mathscr{R}}_{\mathscr{M} l l}\left(U_{1}, \ldots U_{m}\right)- \\
\quad-M \sum_{P}^{\prime \prime} \sum_{a=1}^{A} \mathscr{X}_{\mathscr{M} l l}\left(U_{1 a 1}^{P} \cup \ldots \cup U_{1 a s(a)}^{P}, \ldots U_{1 a r(1)}^{P}\right) \times \\
\quad \times \prod_{j=2}^{k(P)} \mathscr{X}_{\mathscr{M} / l}\left(U_{j 1}^{P}, \ldots U_{j r(j)}^{P}\right) \prod_{\text {conn }} \Delta_{l} .
\end{aligned}
$$

Here $\sum_{P} \prime \prime$ extends over those proper partitions $P$ of $\left\{U_{1}, \ldots U_{m}\right\}$, where $l$ connects two vertices from $U_{11}^{P}, \ldots U_{1 r(1)}^{P}$, and $\sum_{a=1}^{A}$ over all IPI subgraphs $G\left(U_{1 a 1}^{P}, \ldots U_{1 a s(a)}^{P}, \mathscr{M}\right), 1<s(a) \leqq r(1)$, which become IPR without $l$.

It is easy to see that the right hand side of (2.6) and (2.8) coincide:

$$
-M \overline{\mathscr{R}}_{\mathscr{M} / l}\left(U_{1}, \ldots U_{m}\right)
$$

becomes $\mathscr{X}_{\mathscr{M} / l}\left(U_{1}, \ldots U_{m}\right)$, if $G\left(U_{1}, \ldots U_{m}, \mathscr{M} \mid l\right)$ is IPI and otherwise

1 The proof in [1], [2] is incorrect. Unfortunately the erroneous identity $R\left(G_{a_{1}} \ldots G_{a_{k}}: \Gamma^{\prime}\right)=\Delta\left(\Gamma^{\prime}\right) R\left(G_{a_{1}} \ldots G_{a_{k}}\right)$ has been used repeatedly in discussing the analytical properties of the $R$-operation (e.g. [1], (4.4)ff; [1], Satz 4). 
$\mathscr{X}_{\mathscr{M} / l}\left(U_{1} \cup \cdots \cup U_{m}\right)$. By inverting the order of the summations $\sum_{P}^{\prime \prime}$ and $\sum_{a}$ the second term in (2.8) is identified with the sum over all IPI $G\left(U_{j 1}, \ldots U_{j r(j)}, \mathscr{M}\right)$ which are IPR without $l$ and over all proper partitions of $\left\{U_{j 1} \cup \cdots \cup U_{j r(j)}, U_{j(r(j)+1)}, \ldots U_{j m}\right\}$.

This proves Lemma 2.2 and under the same assumptions:

Lemma 2.3:

$$
\begin{aligned}
\overline{\mathscr{R}}_{\mathscr{M}}\left(U_{1}, \ldots U_{m}\right)=\overline{\mathscr{R}}_{\mathscr{M} / l}\left(U_{1}, \ldots U_{m}\right)+ \\
\quad+\sum_{j}^{\prime} \overline{\mathscr{R}}_{\mathscr{M} / l}\left(U_{j 1} \cup \cdots \cup U_{j r(j)}, U_{j(r(j)+1)}, \ldots U_{j m}\right)
\end{aligned}
$$

where $\sum_{j}^{\prime}$ extends over all IPI $G\left(U_{j 1}, \ldots U_{j r(j)}, \mathscr{M}\right), 1<r(j)<m$, which become IPR without $l$.

If we collect sums of iterated $M$-operations appearing in $\mathscr{R}_{\mathscr{L}}\left(V_{1}, \ldots V_{n}\right)$ into non-overlapping sequences of $M$ - and $(1-\mathrm{M})$ operations, the following definition arises:

Def.: Let $G\left(V_{1}, \ldots V_{n}, \mathscr{L}\right)$ be IPI. A tree $T=(\mathfrak{U}, \mathscr{M}, \sigma)$ is a set $\mathfrak{U}$ of generalized vertices, a subset $\mathscr{M} \subset \mathscr{L}$ and a mapping $\sigma: \mathfrak{U} \rightarrow\{-1,0,+1\}$ satisfying (A), (B) and (C):

(A) $\left\{V_{1}, \ldots V_{n}\right\} \in \mathfrak{U} ; V_{i} \in \mathfrak{U}, 1 \leqq i \leqq n$; if $U_{1}, U_{2} \in \mathfrak{U}$, then either $U_{1} \cap U_{2}=\emptyset$ or $U_{1} \subset U_{2}$ or $U_{2} \subset U_{1}$.

Remark: The $U \in \mathcal{U}$ can be uniquely labeled by their position in a chain of elements in $\mathfrak{U}$ :

$$
U\left(i_{0}, \ldots i_{k}\right) \subset U\left(i_{0}, \ldots i_{k-1}\right) \subset \cdots \subset U\left(i_{0}\right)=\left\{V_{1}, \ldots V_{n}\right\},
$$

where $i_{0}=1$ and the $i_{r} \geqq 1$ are integers. We set $I=\left(i_{0}, \ldots i_{k}\right)$ and $\left.(I, i)=i_{0}, \ldots i_{k}, i\right)$ and thus $U(I, i) \subset U(I) . \mathscr{I}=\{I: U(I) \in \mathcal{U}\}$.

(B) Either $U(I)=V_{i}$ for some $1 \leqq i \leqq n$ or $\mathscr{M}$ connects the $\{U(I, i):(I, i) \in \mathscr{I}\}$ either IPI or linearly without closed loops.

Example: Fig. 3.
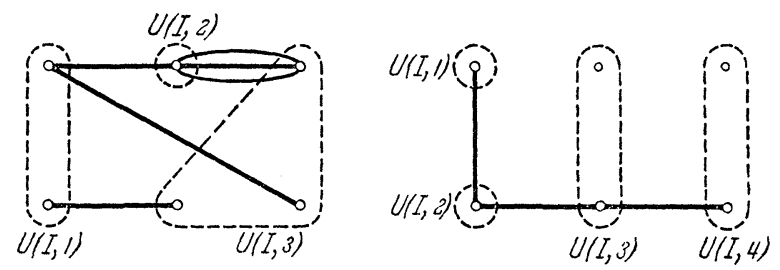

Fig. 3. Connection of the $\{U(I, i)\} \subset U(I)$ by $\mathscr{M}$.

(C) $\sigma\left(U\left(i_{0}\right)\right)=+1 ; \sigma(U(I))=0$, iff $U(I)=V_{i}$ for some $1 \leqq i \leqq n$. If $\sigma(U(I))=-1$, then $G(\{U(I, i)\}, \mathscr{M})$ is IPR. If $G(\{U(I, i)\}, \mathscr{M})$ is IPI, then $\sigma(U(I))=+1$ and $\sigma(U(I, i)) \leqq 0$ for all $(I, i) \in \mathscr{I}$. 
Def.: Let $T=(\mathfrak{U}, \mathscr{M}, \sigma)$ be a tree for a IPI $G\left(V_{1}, \ldots V_{n}, \mathscr{L}\right)$. Then the Feynman amplitude $\mathscr{F}_{T}\left(V_{1}, \ldots V_{n}\right)=\mathscr{F}_{\mathscr{M}}\left(U\left(i_{0}\right)\right)$ is defined recursively from the $\mathscr{F}_{\mathscr{M}}(U(I))$ of the "branches" $U(I) \in \mathcal{U}$ by:

(a) If $U(I)=V_{i}$, then $\mathscr{F}_{\mathscr{M}}(U(I))=1$ and $U(I)$ is called a "twig".

(b) If $\sigma(U(I))=-1$, then $U(I)$ is a "twig" and

$$
\mathscr{F}_{\mathscr{M}}(U(I))=-M\left\{\prod_{(I, i) \in \mathscr{I}} \mathscr{F}_{\mathscr{M}}(U(I, i)) \prod_{\text {conn }} \Delta_{l}\right\} .
$$

(c) If $\sigma(U(I))=+1$ and $G(\{U(I, i)\}, \mathscr{M})$ is IPR, then $U(I)$ is called a "bough" and

$$
\mathscr{F}_{\mathscr{M}}(U(I))=(1-M)\left\{\prod_{(I, i) \in \mathscr{I}^{M}} \mathscr{F}_{\mathscr{M}}(U(I, i)) \prod_{\text {conn }} \Delta_{l}\right\} .
$$

(d) If $\sigma(U(I))=+1$ and $G(\{U(I, i)\}, \mathscr{M})$ is IPI, then $U(I)$ is called a "bud" and

$$
\mathscr{F}_{\mathscr{M}}(U(I))=(1-M) \overline{\mathscr{R}}_{\mathscr{M}}(\{U(I, i)\})
$$

is defined by $(2.2),(2.3)$ starting from the $\mathscr{F}_{\mathscr{M}}(U(I, i))[=1$ or $(2.11)]$ as vertex parts $\mathscr{X}(U(I, i))$.

In (2.11), (2.12) $\prod_{\text {conn }} \Delta_{l}$ extends over all $l \in \mathscr{L}$, which connect different $U(I, i) \subset U(I)$.

Example: If $G\left(V_{1}, \ldots V_{n}, \mathscr{L}\right)$ is IPI, then $\mathscr{R}_{\mathscr{L}}\left(V_{1}, \ldots V_{n}\right)$ $=(1-M) \overline{\mathscr{R}}_{\mathscr{L}}\left(V_{1}, \ldots V_{n}\right)$ is a tree with the bud $\left\{V_{1}, \ldots V_{n}\right\}$ and the twigs $V_{i}, 1 \leqq i \leqq n$. A repeated application of Lemma 2.3 will again lead to sums of trees (see Lemma 2.4).

Def.: The order of $U(I), I=\left(i_{0}, \ldots i_{k}\right)$, is the length $k$ of the chain (2.10). Let $\sigma(U(I))=1$. Then the $b$-order of $U(I)$ is the length of the subchain of (2.10) consisting only of buds and boughs.

Def.: $l$ is contained in $U(I)$, if $V_{i l}, V_{f_{l}} \in U(I)$.

For each $l \in \mathscr{L}$ there exists an $U(I) \in \mathfrak{U}$ of maximal order which contains $l$ : if $V_{i_{l}}=U\left(I^{\prime}\right), V_{i_{l}}=U\left(I^{\prime \prime}\right)$, then $i_{o}^{\prime}=i_{o}^{\prime \prime}$ for $0 \leqq \varrho \leqq r$ and $i_{r+1}^{\prime} \neq i_{r+1}^{\prime \prime}$ for some $r$; then $I=\left(i_{0}^{\prime}, \ldots, i_{r}^{\prime}\right)$. If $\sigma(U(I))=-1$, then the bud or bough of maximal order containing $l$ contains $U(I)$ properly. The following lemma motivates our arborological language:

Lemma 2.4.: Let $G\left(V_{1}, \ldots V_{n}, \mathscr{L}\right)$ be IPI and $l_{1}>\cdots>l_{L}$ be any ordering of the lines $\mathscr{L}$. Then there exists a finite set of trees $T=(\mathfrak{U}, \mathscr{M}, \sigma)$ such that

(a) $\mathscr{R}_{\mathscr{L}}\left(V_{1}, \ldots V_{n}\right)=\sum_{T} \mathfrak{F}_{T}\left(V_{1}, \ldots V_{n}\right)$.

(b) Each $\mathscr{M}$ contains exactly $n-1$ lines.

(c) If $l \in \mathscr{L}-\mathscr{M}$ and if $U\left(i_{0}, \ldots i_{s}\right)$ is the branch and $U\left(i_{0}, \ldots i_{r}\right)$ the bough of maximal order containing $l$, then $l>l^{\prime}$ for all $l^{\prime} \in \mathscr{M}_{1}\left(i_{0}, \ldots i_{e}\right)$, $r \leqq \varrho \leqq s$, where $\mathscr{M}_{1}(I)$ is the set of all $l^{\prime} \in \mathscr{M}$, which are contained in $U(I)$ but in no twig $U\left(I^{\prime}\right) \subset U(I), U\left(I^{\prime}\right) \neq U(I)$. 
Proof: Using the fact that $\mathscr{R}_{\mathscr{L}}\left(V_{1}, \ldots V_{n}\right)$ is a tree and repeatedly the reduction formula (2.9) we shall prove Lemma 2.4 by complete induction with respect to the number of lines in $\mathscr{L}-\mathscr{M}$.

We assume that after $m$ steps, $0 \leqq m<L+1-n, \mathscr{R}_{\mathscr{L}}\left(V_{1}, \ldots V_{n}\right)$ can be represented as a finite sum $\sum \mathscr{F}_{T}\left(V_{1}, \ldots V_{n}\right)$ of trees $T=(\mathfrak{U}, \mathscr{M}, \sigma)$. In each tree every bough is of b-order $\leqq k$ and every bud of b-order $\geqq k$ for some $k \geqq 0$. $\mathscr{M}$ consists of exactly $L-m$ lines. If $l \in \mathscr{L}-\mathscr{M}$ and $U\left(i_{0}, \ldots i_{s}\right)$ is the branch and $U\left(i_{0}, \ldots i_{r}\right)$ the bud or bough of maximal order containing $l$, then $l>l^{\prime}$ for all $l^{\prime} \in \mathscr{M}_{1}\left(i_{0}, \ldots i_{Q}\right)$, $r \leqq \varrho \leqq s$.

Obviously $\mathscr{R}_{\mathscr{L}}\left(V_{1}, \ldots V_{n}\right)$ satisfies all these properties for $m=0$. We shall show that the induction hypothesis remains valid, if in any tree $T$ we reduce one of the buds $U(I)$ of smallest b-order by applying Lemma 2.3 to its Feynman amplitude (2.13) and by reducing with respect to the largest line $l$ in $\mathscr{M}_{1}(I)$. By definition all branches $U(I, i) \subset U(I)$ are twigs. Therefore one obtains

$$
\begin{aligned}
& \mathscr{F}_{\mathscr{M}}(U(I))=(1-M) \overline{\mathscr{R}}_{\mathscr{M} / l}(U(I, 1), \ldots U(I, s))+ \\
& \quad+\sum(1-M) \overline{\mathscr{R}}_{\mathscr{M l l}}\left(U\left(I, j_{1}\right) \cup \cdots \cup U\left(I, j_{r(j)}\right), \ldots U\left(I, j_{s}\right)\right) .
\end{aligned}
$$

$\sum_{j}$ extends over all IPI $G\left(U\left(I, j_{1}\right), \ldots U\left(I, j_{r(j)}\right), \mathscr{M}\right)$, which are IPR without $l$. Let $\left\{U\left(I, h_{i}\right)\right\}, 1 \leqq i \leqq a$, and $\left\{U\left(I, h_{i j}\right), 1 \leqq j \leqq c(i)\right\}$, $1 \leqq i \leqq b$, be the partition of $\left\{U\left(I, j_{1}\right), \ldots U\left(I, j_{r(j)}\right)\right\}$, which characterizes the IPI components of $G\left(U\left(I, j_{1}\right), \ldots U\left(I, j_{r(j)}\right), \mathscr{M} \mid l\right)$. Then the vertex part for $U\left(I, j_{1}\right) \cup \cdots \cup U\left(I, j_{r(j)}\right)$ factorizes by Lemma 2.1 into

$$
\begin{gathered}
\mathscr{X}_{\mathscr{M} l l}\left(U\left(I, j_{1}\right) \cup \cdots \cup U\left(I, j_{r(j)}\right)\right) \\
=-M \overline{\mathscr{R}}_{\mathscr{M l l}}\left(U\left(I, j_{1}\right), \ldots U\left(I, j_{r(j)}\right)\right) \\
=-M\left\{\prod_{i=1}^{a} \mathscr{F}_{\mathscr{M}}\left(U\left(I, h_{i}\right)\right) \times\right. \\
\left.\times \prod_{i=1}^{b}\left[(1-M) \overline{\mathscr{R}}_{\mathscr{M} / l}\left(U\left(I, h_{i 1}\right), \ldots U\left(I, h_{i c}(i)\right)\right)\right] \prod_{\text {conn }} \Delta_{l}\right\} .
\end{gathered}
$$

Similarly, if $G(U(I, 1), \ldots U(I, s), \mathscr{M} / l)$ is IPR, the first term on the right hand side of (2.14) can be decomposed:

$$
\begin{aligned}
&(1-M) \overline{\mathscr{R}}_{\mathscr{M l l}}(U(I, 1), \ldots U(I, s)) \\
&=(1-M)\left\{\prod_{i=1}^{d} \mathscr{F}_{\mathscr{M}}\left(U\left(I, k_{i}\right)\right) \times\right. \\
&\left.\times \prod_{i=1}^{e}\left[(1-M) \overline{\mathscr{R}}_{\mathscr{M} l l}\left(U\left(I, k_{i 1}\right), \ldots U\left(I, k_{i f(i)}\right)\right)\right] \prod_{\text {conn }} \Delta_{\imath}\right\} .
\end{aligned}
$$

On the other hand all $G\left(U\left(I, j_{1}\right) \cup \cdots \cup U\left(I, j_{r(j)}\right), \ldots U\left(I, j_{s}\right), \mathscr{M}(l)\right.$ are IPI in a bud $U(I)$, and the corresponding Feynman amplitude does not factorize beyond (2.14). 
It is easy to see that only in $\mathscr{F}_{\mathscr{M}}(U(I))$ the reduction of $\mathscr{M}$ to $\mathscr{M} / l$ has the effect of introducing new branches corresponding to a rearrangement of counter terms. By definition $\mathscr{M}$ connects linearly the $\left\{U\left(I^{\prime}, i\right)\right\}$ in a bough or twig $U\left(I^{\prime}\right)$, while in a bud $G\left(\left\{U\left(I^{\prime}, i\right)\right\}, \mathscr{M}\right)$ is IPI and $\mathscr{M}$ determines the counter terms in $(1-M) \overline{\mathscr{R}}_{\mathscr{M}}\left(\left\{U\left(I^{\prime}, i\right)\right\}\right)$ by (2.2).

Now, the bud $U(I)$ in (2.14) was chosen to lie in no other bud. Therefore all branches containing $U(I)$ are boughs or twigs, whose sets of connecting lines in $\mathscr{M}$ is unaffected by omitting any $l \in \mathscr{M}$ contained in $U(I)$. For the same reason $\mathscr{M}$ and $\mathscr{M} / l$ are equivalent in branches $U\left(I^{\prime}\right) \cap U(I)=\emptyset$. Lastly every $U\left(I^{\prime}\right) \subset U(I)$ is contained in some $U(I, i)$, and we can also here replace $\mathscr{M}$ by $\mathscr{M} / l$, since $l$ was chosen not to lie in any $U(I, i)$.

Thus if we insert (2.14) into the recursive definition of $\mathscr{F}_{T}\left(V_{1}, \ldots V_{n}\right)$, the contribution of each summand defines a new tree $T^{\prime}=\left(\mathfrak{U}^{\prime}, \mathscr{M}^{\prime}, \sigma^{\prime}\right), \mathscr{M}^{\prime}$ $=\mathscr{M} / l$, if one separates the twigs and buds in (2.15), (2.16). Since the bud $U(I)$ was of minimal b-order, any bough arising from (2.14), if $G(\{U(I, i)\}, \mathscr{M} \mid l)$ is IPR, lies outside of all buds, and every bud in $T^{\prime}$ is of $\mathrm{b}$-order $\geqq k^{\prime}, k^{\prime}=k$ or $k+1$.

It remains to check the order relations in $\left(\mathfrak{U}^{\prime}, \mathscr{M}^{\prime}, \sigma^{\prime}\right)$ between the lines in $\mathscr{M}^{\prime}=\mathscr{M} \mid l$ and $\mathscr{L}-\mathscr{M}^{\prime}$. Consider the line $l$. The bough or bud $U$ of maximal order containing $l$ is either $U(I)$ or one of the new buds $U\left(I, h_{i 1}\right) \cup \cdots \cup U\left(I, h_{i c(i)}\right)$ in (2.15) or $U\left(I, k_{i 1}\right) \cup \cdots \cup U\left(I, k_{i f(i)}\right)$ in (2.16), and $l$ does not lie in any twig contained in $U$, since each of these twigs is contained in one of the $U(I, i)$. Therefore the lines in $\mathscr{M} / l$, which lie in $U$, but in no twig in $U$, are a subset of $\mathscr{M}_{1}(I)$, from which $l$ was chosen as the largest line.

Consider now an $l^{\prime} \in \mathscr{L}-\mathscr{M}$, which is not contained in $U(I)$. Since in all $\left(\mathfrak{U}^{\prime}, \mathscr{M}^{\prime}, \sigma^{\prime}\right)$ the structure of the branches outside of $U(I)$ is the same as in $(\mathcal{U}, \mathscr{M}, \sigma)$ and since the set of $l^{\prime \prime} \in \mathscr{M}^{\prime}$, which lie in $U(I)$ but in no twigs in $U(I)$, is a subset of $\mathscr{M}_{1}(I)$, the order relations hold for a fortiori for $l^{\prime}$.

Suppose finally that $l^{\prime} \in \mathscr{L}-\mathscr{M}$ is contained in $U(I)$. Then the bud or bough $U$ of maximal order containing $l^{\prime}$ satisfies one of the following alternatives:

a) $U$ is one of the buds of $(\mathcal{U}, \mathscr{M}, \sigma)$ contained in some $U(I, i)$. As the reduction did not change the structure inside of the $U(I, i)$, the order relations are preserved.

b) $U$ is one of the newly created buds in (2.15) or (2.16). Then the twigs in $U$ are contained in the $U(I, i)$. Thus $l^{\prime \prime} \in \mathscr{M}_{1}(I)$ for all $l^{\prime \prime} \in \mathscr{M}^{\prime}$, which lie in $U$ but in no twig contained in $U$. If $l^{\prime}$ lies outside of all twigs in $U$, then by the order relations in $(\mathcal{U}, \mathscr{M}, \sigma) l^{\prime}>l^{\prime \prime}$ for all $l^{\prime \prime} \in \mathscr{M}_{1}(I)$. If the branch $U^{\prime}$ of maximal order containing $l^{\prime}$ is different from $U$, then the chain of twigs between $U^{\prime}$ and $U$ is contained in some 
$U(I, i): U^{\prime} \subset U^{\prime \prime} \subset \cdots \subset U(I, i) \subset U$. In the chain $U^{\prime} \subset U^{\prime \prime} \subset \cdots \subset U(I, i)$ the order relations of $(\mathfrak{U}, \mathscr{M}, \sigma)$ continue to hold, and as before $l^{\prime}$ satisfies the order relations in $U$.

c) $U=U(I)$. Then it is possible that the twig $U^{\prime}$ of maximal order containing $l^{\prime}$ generates a chain of twigs $U^{\prime} \subset \cdots \subset U(I, i) \subset U^{\prime \prime} \subset U(I)$, where $U^{\prime \prime}$ is one of the twigs $U\left(I, j_{1}\right) \cup \cdots \cup U\left(I, j_{r(j)}\right)$ in (2.14). Again in $U^{\prime} \subset \cdots \subset U(I, i)$ the order relations are not affected by going from $\mathscr{M}$ to $\mathscr{M} / l$, while in $U^{\prime \prime}$ or $U(I)$ the critical lines belong to $\mathscr{M}_{1}(I)$.

This completes the induction. After $L+1-n$ reductions one has eliminated all buds in all trees, and one arrives at the statement of Lemma 2.4 .

Example: Consider the graph $G\left(V_{1}, \ldots V_{4}, \mathscr{L}\right)$ (Fig. 4).

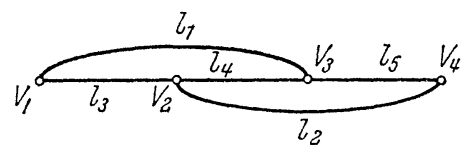

Fig. 4. $G\left(V_{1}, \ldots V_{4}, \mathscr{L}\right)$

For $l_{1}>\cdots>l_{5} \mathscr{R}_{\mathscr{L}}\left(V_{1}, \ldots V_{4}\right)$ leads to the trees (Fig. 5). Here twigs are denoted by a dotted, boughs by a continuous encircling. The lines in $\mathscr{M}$ are solidly marked. In the case $l_{4}>l_{3}>l_{5}>l_{1}>l_{2}$ one obtains Fig. 6.

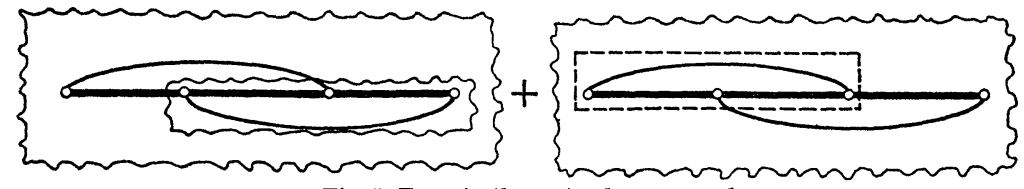

Fig. 5. Trees in the sector $l_{1}>\cdots>l_{5}$

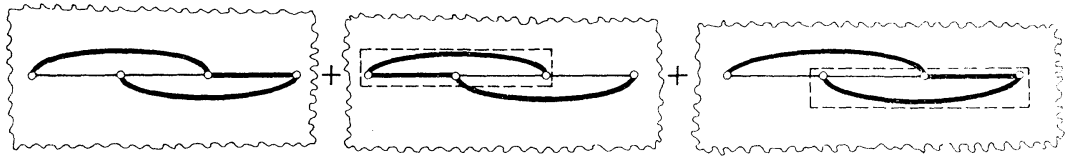

Fig. 6. Trees in the sector $l_{4}>l_{3}>l_{5}>l_{1}>l_{2}$

\section{Analytical properties of the $\mathscr{R}$-operation}

In this section we shall determine the analytical structure of the $\mathscr{R}$-operation in $p$-space. For $\varepsilon>0, r>0$ we first carry out all convolution integrals in $p$-space and study the remaining $\alpha$-integrands. The essential simplification occurs after reducing in every sector

$$
\alpha_{l_{1}} \geqq \cdots \geqq \alpha_{l_{L}} \geqq r
$$

the $\alpha$-integrands of $\mathscr{R}_{\mathscr{L}}\left(V_{1}, \ldots V_{n}\right)$ into a sum of trees with respect to the order relation $l_{1}>\cdots>l_{L}$. 
For a tree $(\mathfrak{U}, \mathscr{M}, \sigma)$ we define:

$\mathscr{V}_{0}(I)=\left\{V_{i} \in U(I)\right\}$

$\mathscr{L}_{0}(I)=\left\{l \in \mathscr{L}: V_{i_{l}}, V_{f_{l}} \in U(I)\right\}$

$\mathscr{L}(I)=\{l \in \mathscr{L}:$ connecting different $U(I, i) \subset U(I)\}$

$\mathscr{M}(I)=\{l \in \mathscr{M}:$ connecting different $U(I, i) \subset U(I)\}$

$\mathscr{M}_{0}(I)=\left\{l \in \mathscr{M}: V_{i_{i}}, V_{f_{l}} \in U(I)\right\}$

$\mathscr{M}_{1}(I)=\left\{l \in \mathscr{M}:\right.$ contained in $U(I)$ but in no twig $U\left(I^{\prime}\right) \subset U(I)$,

$$
\left.U\left(I^{\prime}\right) \neq U(I)\right\}
$$

$\mathfrak{U}_{1}(I)=\left\{U\left(I^{\prime}\right) \subset U(I)\right.$ : not contained in any twig $U\left(I^{\prime \prime}\right) \subset U(I)$,

$$
\left.U\left(I^{\prime \prime}\right) \neq U(I)\right\} \text {. }
$$

The number of elements in any of these sets $\mathscr{N}$ is denoted by $|\mathscr{N}|$, e.g., $\left|\mathscr{L}_{0}\left(i_{0}\right)\right|=L . I \geqq I^{\prime}$, if $U(I) \supset U\left(I^{\prime}\right)$, and $I>I^{\prime}$, if in addition $U(I) \neq$ $\neq U\left(I^{\prime}\right)$. The superficial divergence $(1.7)$ of $G\left(\mathscr{V}_{0}(I), \mathscr{L}\right)$ becomes

$$
v(I)=\sum_{l \in \mathscr{L}_{0}(I)}\left(r_{l}+2\right)-4\left(\left|\mathscr{V}_{0}(I)\right|-1\right)
$$

Lemma 3.1: Let $T=(\mathcal{U}, \mathscr{M}, \sigma)$ be a tree in the decomposition of $\mathscr{R}_{\mathscr{L}}\left(V_{1}, \ldots V_{n}\right)$ in (3.1). Then the Feynman amplitude $\mathscr{F}_{\mathscr{M}}(U(I))$ of any bough $U(I) \in \mathcal{U}$ is for fixed $\alpha$ a finite sum of terms of the form

$$
\begin{aligned}
& \delta\left(\sum_{V_{i} \in \mathscr{V}_{0}(I)} p_{i}\right) \int_{0}^{1} \cdots \int_{0}^{1}\left[\underset{\substack{I^{\prime} \leqq I \\
\sigma\left(I^{\prime}\right)=1}}{\prod} d \tau\left(I^{\prime}\right)\right] P^{\mathrm{I}}(p) Q^{\mathrm{I}}(\alpha, \tau) \times \\
& \times\left[\prod_{I^{\prime} \leqq I} D^{I^{\prime}} R^{I^{\prime}}\left(A^{I^{\prime}}\right) S^{I^{\prime}}\left(B^{I^{\prime}}\right)\right] \times \\
& \times \exp \left[i \sum_{V_{i}, V_{j} \in \mathscr{V}_{0}(I)} \tau(I)^{2} A_{i j}^{\mathrm{I}} p_{i} p_{j}-i \sum_{l \in \mathscr{L}_{0}(I)} \alpha_{l}\left(m_{l}^{2}-i \varepsilon\right)\right] .
\end{aligned}
$$

If $\sigma(U(I))=-1$, then $\mathscr{F}_{\mathscr{M}}(U(I))$ has the same structure (3.4) except for $\sum \tau(I)^{2} A_{i j}^{\mathrm{I}} p_{i} p_{j}$ being replaced by zero. The integrand in (3.4) satisfies:

(1) $P^{\mathrm{I}}(p)$ is a monomial in $p_{i}, V_{i} \in \mathscr{V}_{0}(I)$, of degree $2 x(I)+z(I)$.

(2) $Q^{\mathrm{I}}(\alpha, \tau)$ is a rational function in $\alpha_{l}, l \in \mathscr{L}_{0}(I)$ and $\tau\left(I^{\prime}\right), I^{\prime} \leqq I$, homogeneous of degree 0 in $\alpha$ and uniformly bounded in (3.1) for $r \geqq 0$ and all $0 \leqq \tau\left(I^{\prime}\right) \leqq 1$.

(3) $D^{\mathrm{I}^{\prime}}=\prod_{l \in \mathscr{L}\left(I^{\prime}\right)-\mathscr{M}} D_{l}^{-2}$, where $D_{l}=D_{l}(\alpha, \tau)$ is rational in $\alpha, \tau$, homogeneous of degree +1 in $\alpha$ and $D_{l} \geqq \alpha_{l}$ for $r \geqq 0$.

(4) $A^{\mathrm{I}^{\prime}}=\left(A_{i j}^{\mathrm{I}^{\prime}}\right)$ is a positive semidefinite quadratic form. The $A_{i j}^{\mathrm{I}^{\prime}}(\alpha, \tau)$ are rational in $\alpha, \tau$, homogeneous of degree +1 in $\alpha$ and satisfy uniformly for $r \geqq 0$

$$
\left|A_{i j}^{I^{\prime}}\right| \leqq c\left(I^{\prime}\right) \max \left\{\alpha_{l}: l \in \mathscr{M}_{1}\left(I^{\prime}\right)\right\}, c\left(I^{\prime}\right)<\infty .
$$

(5) $R^{\mathrm{I}^{\prime}}\left(A^{\mathrm{I}^{\prime}}\right)$ is a monomial of degree $x\left(I^{\prime}\right)$ in $A_{i j}^{\mathrm{I}^{\prime}}, V_{i}, V_{j} \in \mathscr{V}_{0}\left(I^{\prime}\right)$.

(6) $B_{i j}^{I \prime}(\alpha, \tau)$ is rational in $\alpha, \tau$, homogeneous of degree -1 in $\alpha$, and satisfies uniformly in (3.1) for $r \geqq 0$

$$
\left|B_{i j}^{I^{\prime}}\right|<d\left(I^{\prime}\right) \max \left\{\alpha_{l}^{-1}, l \in \mathscr{L}\left(I^{\prime}\right)-\mathscr{M}\right\}, d\left(I^{\prime}\right)<\infty \text {. }
$$


(7) $S^{1^{\prime}}\left(B^{\mathrm{I}^{\prime}}\right)$ is a monomial of degree $y\left(I^{\prime}\right)$ in the $B_{i j}^{\mathrm{I}^{\prime}}, \quad 1 \leqq i$, $j \leqq\left|\mathscr{V}_{0}\left(I^{\prime}\right)\right|+\left|\mathscr{L}\left(I^{\prime}\right)\right|-\left|\mathscr{M}\left(I^{\prime}\right)\right|$.

(8) $x\left(I^{\prime}\right), y\left(I^{\prime}\right), z\left(I^{\prime}\right) \geqq 0$ are integers satisfying:

$$
\begin{gathered}
x\left(I^{\prime}\right)=y\left(I^{\prime}\right)=z\left(I^{\prime}\right)=0, \text { for } \sigma\left(U\left(I^{\prime}\right)\right)=0 \\
x\left(I^{\prime}\right) \geqq\left[\left[\frac{v\left(I^{\prime}\right)+1-z\left(I^{\prime}\right)}{2}\right]\right], \text { for } \sigma\left(U\left(I^{\prime}\right)\right)=1 \\
x\left(I^{\prime}\right) \leqq \frac{v\left(I^{\prime}\right)-z\left(I^{\prime}\right)}{2} \text { for } \sigma\left(U\left(I^{\prime}\right)\right)=-1 \\
2 y\left(I^{\prime}\right)+z\left(I^{\prime}\right) \leqq \sum_{l \in \mathscr{L}(I)} r_{l}+\sum_{\left(I^{\prime}, i\right) \in \mathscr{I}}\left[2 x\left(I^{\prime}, i\right)+z\left(I^{\prime}, i\right)\right],
\end{gathered}
$$

where $[[k]]$ is the smallest integer $\geqq k$.

Remark: This theorem sharpens the statements in [1], Satz 4. The representation of the renormalized amplitudes as sums of terms of the form (3.4), each of which being locally $\alpha$-integrable in the whole range $0 \leqq \alpha_{l}<\infty, 1 \leqq l \leqq L$, as stated in [2],(8.3), (8.4), has not been proved.

Proof: Let $s(I)$ be the length of the longest chain of branches $U(I) \supset U\left(I_{1}\right) \supset \cdots \supset U\left(I_{s(I)}\right)$ (with proper inclusion) contained in $U(I)$. We shall prove Lemma 3.1 by induction on $s(I)$, starting from an original vertex with $s(I)=0$. As induction assumption in the case $s(I)>0$ we take Lemma 3.1 for granted for all $U\left(I^{\prime}\right)$ with $s\left(I^{\prime}\right)<s(I)$ and thus for all $U\left(I^{\prime}\right) \subset U(I)$.

We compute $\mathscr{F}_{\mathscr{M}}(U(I))$ in $p$-space using $(2.11)$ or (2.12). Then the $p$-dependent part of $\mathscr{F}_{\mathscr{M}}(U(I, 1)) \mathscr{F}_{\mathscr{M}}(U(I, 2)) \Delta_{l_{12}}$ becomes for $\sigma(U(I, 1))=\sigma(U(I, 2))=+1$ a sum of terms

$$
\begin{gathered}
\int d k \delta\left(\sum_{V_{i} \in \mathscr{V}_{0}(I, 1)}\left(p_{i}+e_{i} k\right)\right) \delta\left(\sum_{V_{j} \in \mathscr{V}_{0}(I, 2)}\left(p_{j}+e_{j} k\right)\right) P_{l_{12}}(k) \\
P^{(I, 1)}\left(\left\{p_{i}+e_{i} k\right\}\right) P^{(I, 2)}\left(\left\{p_{j}+e_{j} k\right\}\right) \\
\exp \left[i \alpha_{l_{12}} k^{2}+i \tau(I, 1)^{2} \sum_{i, i^{\prime}} A_{i i^{\prime}}^{(I, 1)}\left(p_{i}+e_{i} k\right)\left(p_{i^{\prime}}+e_{i^{\prime}} k\right)+\right. \\
\left.+i \tau(I, 2)^{2} \sum_{j, j^{\prime}} A_{j j^{\prime}}^{(I, 2)}\left(p_{j}+e_{j} k\right)\left(p_{j^{\prime}}+e_{j^{\prime}} k\right)\right],
\end{gathered}
$$

where $e_{i}=+1$ for $i=i_{l_{12}}, e_{i}=-1$ for $i=f_{l_{12}}$ and 0 otherwise. If $U(I, 1)$ and $U(I, 2)$ are not both boughs, one obtains similar terms. After carrying out the $k$-integration with the help of one of the $\delta$-functions $(3.10)$ becomes a sum of terms

$$
\delta\left(\sum_{V_{i} \in \mathscr{V}_{0}(I, 1) \cup \mathscr{V}_{0}(I, 2)} p_{i}\right) P^{\prime}(p) \exp \left[i \sum A_{i j}^{\prime} p_{i} p_{j}\right],
$$

where $P^{\prime}(p)$ is a monomial in $p_{i}, V_{i} \in \mathscr{F}_{0}(I, 1) \cup \mathscr{F}_{0}(I, 2)$, of degree $\leqq r_{l_{12}}+\sum_{i=1}^{2}[2 x(I, i)+z(I, i)]$ and $A^{\prime}$ is a positive semidefinite quadratic form, whose coefficients $A_{i j}^{\prime}$ are linear combinations of the $A_{i i^{\prime}}^{(I, 1)} \tau(I, 1)^{2}, A_{j j^{\prime}}^{(I, 2)} \tau(I, 2)^{2}$ and $\alpha_{l_{12}}$. By a similar argument one obtains for

$$
\prod_{(I, i) \in \mathscr{I}} \mathscr{F}_{\mathscr{M}}(U(I, i)) \prod_{l \in \mathscr{M}(I)} \Delta_{l}
$$


in $p$-space a sum of terms

$$
\begin{aligned}
& \delta\left(\sum_{V_{i} \in \mathscr{V}(I)} p_{i}\right) \int_{0}^{1} \ldots \int_{0}^{1} \prod_{I^{\prime}<I} d \tau\left(I^{\prime}\right) \prod_{I^{\prime}<I} D^{I^{\prime}} R^{I^{\prime}}\left(A^{I^{\prime}}\right) S^{I^{\prime}}\left(B^{I^{\prime}}\right) \times \\
\times & \prod_{(I, i) \in \mathscr{I}} Q^{(I, i)}(\alpha, \tau) P^{(r)}(p) \exp \left[i \sum A_{i j}^{(r)} p_{i} p_{j}-i \sum \alpha_{l}\left(m_{l}^{2}-i \varepsilon\right)\right],
\end{aligned}
$$

where $P^{(r)}(p)$ is a monomial in the $p_{i}, V_{i} \in \mathscr{F}_{0}(I)$, of degree $\leqq \sum_{l \in \mathscr{M}(I)} r_{l}+$ $+\sum_{(I, i) \in \mathscr{I}}[2 x(I, i)+z(I, i)]$ and $A_{i j}^{(r)}$ is a linear combination of the $\alpha_{l}$, $l \in \mathscr{M}(I)$, and the $A_{i j}^{(I, i)} \tau(I, i)^{2}, \sigma(I, i)=1$. Following [1] we use $p=-\left.i \frac{\partial}{\partial r} \exp [i p r]\right|_{r=0}$ and obtain

$$
P^{(r)}(p)=\left.P^{(r)}(-i \nabla) \exp \left[i \sum_{V_{j} \in \mathscr{V}_{0}(I)} p_{j} r_{j}\right]\right|_{r=0} .
$$

Let us assume that after having incorporated a set $\mathscr{N}$ of $n$ lines, $r \leqq n<\mathscr{L}(I)$, we hav in $p$-space instead of

$$
P^{(r)}(p) \exp \left[i \sum A_{i j}^{(r)} p_{i} p_{j}-i \sum \alpha_{l}\left(m_{l}^{2}-i \varepsilon\right)\right]
$$

in (3.13) a sum of terms

$$
\begin{aligned}
\prod_{l \in \mathscr{N}-\mathscr{M}} D_{l}^{-2} p^{(n)} & (-i D) \exp \left[i \sum_{V_{i}, V_{j} \in \mathscr{V}_{0}(I)} A_{i j}^{(n)} p_{i} p_{j}+i \sum_{k, l=1}^{n} B_{k l}^{(n)} r_{k} r_{l}+\right. \\
& \left.+i \sum_{V_{i} \in \mathscr{V}_{0}(I)} \sum_{k=1}^{n} C_{i k}^{(n)} p_{i} r_{l}-i \sum_{l \in \mathscr{N}} \alpha_{l}\left(m_{l}^{2}-i \varepsilon\right)\right]\left.\right|_{r=0}
\end{aligned}
$$

where $D_{l}$ satisfies (3), $P^{(n)}(-i \nabla)$ is a monomial in the $\frac{\partial}{\partial r_{i}}, 1 \leqq i \leqq n$, of degree

$$
\leqq \sum_{(I, i) \in \mathscr{I}}[2 x(I, i)+z(I, i)]+\sum_{l \in \mathscr{N}} r_{l},
$$

$A^{(n)}$ satisfies (5), $B^{(n)}$ satisfies (7) and $C_{i k}^{(n)}(\alpha, \tau)$ is rational in the $\alpha, \tau$, homogeneous of degree 0 in the $\alpha$ and uniformly bounded in (3.1) for $r \geqq 0$.

We now incorporate another $\Delta_{l^{\prime}}\left(x_{i_{l^{\prime}}}-x_{f^{\prime}}\right)$, where $l^{\prime} \in \mathscr{L}(I)-\mathscr{N}$. Let $e_{i}=+1$ for $i=i_{l^{\prime}}, e_{i}=-1$ for $i=f_{l^{\prime}}$ and $e_{i}=0$ otherwise. For the convolution in $p$-space we have to integrate over $k \in R^{4}$

$$
P_{l^{\prime}}\left(-i \frac{\partial}{\partial r_{n+1}}\right) \exp \left[i \alpha_{l^{\prime}}\left(k^{2}-m_{l^{\prime}}^{2}+i \varepsilon\right)+i r_{n+1} k\right]_{r_{n+1}}=0
$$

multiplied with an expression similar to (3.16) but with $p_{i}$ replaced by $p_{i}+e_{i} k$. Then $(3.16)$ times $P_{l^{\prime}}\left(-i \frac{\partial}{\partial r_{n+1}}\right) \exp \left[-i \alpha_{l^{\prime}}\left(m_{l^{\prime}}^{2}-i \varepsilon\right)\right]$ operates on

$$
\begin{gathered}
\int d k \exp \left[i\left(\alpha_{l^{\prime}}+\sum A_{i j}^{(n)} e_{i} e_{j}\right) k^{2}+i\left(2 \sum A_{i j}^{(n)} p_{i} e_{j}+\right.\right. \\
\left.\left.+\sum C_{i k}^{(n)} e_{i} r_{k}+r_{n+1}\right) k\right]_{r=0} .
\end{gathered}
$$

The Gaussian integral (3.19) can be evaluated by using the identity for 
$a>0$ :

$$
\lim _{\eta \downarrow 0} \int d k \exp \left[i a k^{2}+i p k-\eta\left(k_{0}^{2}+k_{n}^{2}\right)\right]=\frac{\pi^{2}}{i a^{2}} \exp \left[-i \frac{p^{2}}{4 a}\right] .
$$

Then one obtains again an expression of the form (3.16) with $\prod_{\mathscr{N} \rightarrow \mathscr{M}} D_{l}^{-2}$ multiplied by $D_{l^{\prime}}^{-2}$, where

Furthermore

$$
D_{l^{\prime}}=\left(\alpha_{l^{\prime}}+\sum A_{i j}^{(n)} e_{i} e_{j}\right) .
$$

$$
\begin{gathered}
P^{(n+1)}(-i \nabla)=P^{(n)}(-i \nabla) P_{l^{\prime}}\left(-i \frac{\partial}{\partial r_{n+1}}\right) \\
A_{i i^{\prime}}^{(n+1)}=A_{i i^{\prime}}^{(n)}-D_{l^{\prime}}^{-1}\left[\sum_{j j^{\prime}} A_{i j}^{(n)} e_{j} A_{i^{\prime} j^{\prime}}^{(n)} e_{j^{\prime}}\right] \\
B_{k k^{\prime}}^{(n+1)}=B_{k k^{\prime}}^{(n)}-\frac{1}{4} D_{l^{\prime}}^{-1}\left[\sum_{i, i^{\prime}} C_{i k}^{(n)} e_{i} C_{i^{\prime} k^{\prime}}^{(n)} e_{i^{\prime}}\right], 1 \leqq k, k^{\prime} \leqq n, \\
B_{n+1, k}^{(n+1)}=B_{k, n+1}^{(n+1)}=\frac{1}{4} D_{l^{\prime}}^{-1}\left[\sum_{i} C_{i k}^{(n)} e_{i}\right] \\
B_{n+1, n+1}^{(n+1)}=-\frac{1}{4} D_{l^{\prime}}^{-1} \\
C_{i k}^{(n+1)}=C_{i k}^{(n)}-D_{l^{\prime}}^{-1}\left[\sum_{j, j^{\prime}} A_{i j}^{(n)} e_{j} C_{j^{\prime} k}^{(n)} e_{j^{\prime}}\right], 1 \leqq k \leqq n, \\
C_{i, n+1}^{(n+1)}=-D_{l^{\prime}}^{-1} \sum_{j} A_{i j}^{(n)} e_{j} .
\end{gathered}
$$

Since $A^{(n)}$ is positive semidefinite, $D_{l^{\prime}} \geqq \alpha_{l^{\prime}}$ satisfies again (3). Since $l^{\prime} \in \mathscr{L}(I)-\mathscr{M}, \alpha_{l^{\prime}} \geqq \alpha_{l}$ for all $l \in \mathscr{M}_{1}(I)$ by Lemma 2.4. As $A_{i j}^{(n)}$ is majorized by (3.5) for some $c_{n}<\infty$ and $D_{l^{\prime}} \geqq \alpha_{l^{\prime}}, D_{l^{\prime}}^{-1} A_{i j}^{(n)}$ is uniformly bounded in (3.1) for $r \geqq 0$ and $A_{i j}^{(n+1)}$ satisfies (5). The positive semidefiniteness of $A^{(n+1)}$ follows from the inequality

$$
\sum_{i, j} A_{i j}^{(n+1)} x_{i} x_{j} \geqq D_{l^{\prime}}^{-1}\left[\sum_{i, i^{\prime}, j, j^{\prime}} A_{i j}^{(n)} x_{i} x_{j} A_{i^{\prime} j^{\prime}}^{(n)} e_{i^{\prime}} e_{j^{\prime}}-\left(\sum_{i, j} A_{i j}^{(n)} x_{i} e_{j}\right)^{2}\right] \geqq 0 .
$$

Similarly one checks that $B_{k l}^{(n+1)}$ satisfies (7) and that the $C_{i k}^{(n+1)}$ are uniformly bounded in (3.1) for $r \geqq 0$, rational in $\alpha, \tau$, and homogeneous of degree 0 in $\alpha$. Therefore $\prod \mathscr{F}_{\mathscr{M}}(U(I, i)) \prod_{\mathscr{L}(I)} \Delta_{l}$ has in $p$-space the form (3.13) with (3.16) and $\mathscr{N}=\mathscr{L}(I)-\mathscr{L}(I), n=|\mathscr{L}(I)|$ inserted instead of $P^{(r)} \exp [\ldots]$. We set $A_{i j}^{\mathrm{I}}=A_{i j}^{(n)}, B_{i k}^{\mathrm{I}}=B_{i k}^{(n)}, D^{\mathrm{I}}=\prod_{\mathscr{L}(I)-\mathscr{M}^{\prime}} D_{l}^{-2}$.

Finally we carry out the $r$-differentiation. The only terms which survive after setting all $r_{k}=0$, have in $(3.13) P^{(n)}(-i \nabla)$ replaced by a sum of terms

$$
Q(\alpha, \tau) P(p) S^{I}\left(B^{I}\right),
$$

where $Q$ is a polynomial in the $C_{i k}^{(n)}, P(p)$ a monomial in the $p_{i}, V_{i} \in \mathscr{V}_{0}(I)$, of degree $z(I)$ and $S^{\mathrm{I}}\left(B^{\mathrm{I}}\right)$ a polynomial in the $B_{k l}^{\mathrm{I}}$ of degree $y(I)$ with

$$
2 y(I)+z(I) \leqq \sum_{(I, i) \in \mathscr{I}}[2 x(I, i)+y(I, i)]+\sum_{l \in \mathscr{L}_{(I)}} r_{l} .
$$


The inequality (3.28) holds, since every $r$-differentiation of

$$
\exp \left[i \sum B_{k l} r_{k} r_{l}+i \sum C_{i k} p_{i} r_{k}\right]
$$

brings down factors like $B_{k l} r_{k}$ and $C_{i k} p_{i}$. Each $B_{k l} r_{k}$ has to be differentiated again in order to survive for $r_{l k} \rightarrow 0$. Thus $\operatorname{deg} P(p)+2 \operatorname{deg} S^{\mathrm{I}}\left(B^{\mathrm{I}}\right)$ $=z(I)+2 y(I) \leqq \operatorname{deg} P^{(n)}$.

Let $\sigma(U(I))=-1$. If we apply the operation $(-M)$, we obtain instead of $Q(\alpha, \tau) P(p) S^{\mathrm{I}}\left(B^{\mathrm{I}}\right) \exp \left[i \sum A_{i j}^{\mathrm{I}} p_{i} p_{j}\right]$ a sum of terms of the form

$$
Q^{\mathrm{I}}(\alpha, \tau) P^{\mathrm{I}}(p) R^{\mathrm{I}}\left(A^{\mathrm{I}}\right) S^{\mathrm{I}}\left(B^{\mathrm{I}}\right)
$$

Here $P^{\mathrm{I}}(p)$ satisfies $(1)$, and is zero for $z(I)>v(I)$ and otherwise of degree

$$
2 x(I)+z(I) \leqq v(I) \text {. }
$$

Furthermore $Q^{\mathrm{I}}$ satisfies (2); $R^{\mathrm{I}},(4)$; and $S^{\mathrm{I}},(6)$. In the case $\sigma(U(I))=+1$ we use for the remainder of the Taylor series of $f\left(x_{1}, \ldots x_{m}\right)$ around $(0, \ldots 0)$ up to order $n$ the expression

$$
\frac{1}{n !} \int_{0}^{1} d \tau(1-\tau)^{n} \frac{\partial^{n+1}}{\partial \tau^{n+1}} f\left(\tau x_{1}, \ldots \tau x_{m}\right) .
$$

This leads to (3.4) with $x(I) \geqq\left[\left[\frac{v(I)-z(I)+1}{2}\right]\right]$. Q.E.D.

Lemma 3.2: Let $\mathscr{R}_{\mathscr{L}}\left(V_{1}, \ldots V_{n}\right)=\sum_{T^{\prime}} \mathscr{F}_{T}\left(V_{1}, \ldots V_{n}\right)$ hold as above in (3.1). Then the $\alpha$-integrand of every $\mathscr{F}_{T}\left(V_{1}, \ldots V_{n}\right)$ is, together with all $p$-derivatives, absolutely integrable for $r \downarrow 0$.

Proof: Let $\lambda=(2 L)^{-1}$. If we use Lemma 3.1 for $\mathscr{F}_{\mathscr{M}}(U(I))$, then

$$
\prod_{l \in \mathscr{L}_{0}(I)-\mathscr{M}} D_{l}^{\lambda-1} \prod_{I^{\prime} \leqq I} \prod_{l \in \mathscr{M}\left(I^{\prime}\right)} \alpha_{l}^{\lambda-1}
$$

is locally integrable in (3.1) for $r \downarrow 0$. As $\exp \left[-i \sum \alpha_{l}\left(m_{l}^{2}-i \varepsilon\right)\right]$ is strongly decreasing at infinity for $\varepsilon>0$, we have only to show that in every bough $U(I)$

$$
\prod_{I^{\prime} \leqq I}\left[\prod_{l \in \mathscr{L}\left(I^{\prime}\right)-\mathscr{M}} D_{l}^{-(1+\lambda)}\right]\left[\prod_{l \in \mathscr{M}\left(I^{\prime}\right)} \alpha_{l}^{(1-\lambda)}\right] R^{\mathrm{I}^{\prime}}\left(A^{\mathrm{I}^{\prime}}\right) S^{\mathrm{I}^{\prime}}\left(B^{\mathrm{I}^{\prime}}\right)=T^{\mathrm{I}}
$$

remains continuous and bounded for $r \downarrow 0$.

We use induction with respect to the length $s(I)$ of the longest chain of branches contained properly in $U(I)$ and make the following assumption for any $U(I), 0 \leqq s(I)<s$ :

(1) If $\sigma(U(I))=0$, then $T^{\mathrm{I}}=1$.

(2) If $\sigma(U(I))=+1$, then (3.33) is of the form

$$
F^{\mathrm{I}}(\alpha, \tau) G^{\mathrm{I}}(\alpha, \tau)
$$

where $F^{\mathrm{I}}$ and $G^{\mathrm{I}}$ are tempered continuous functions in (3.1) for $r \downarrow 0$. $F^{I}$ is homogeneous in $A_{i j}^{\mathrm{I}}, U\left(I^{\prime}\right) \in \mathcal{U}_{1}(I)$ and $\alpha_{l}, l \in \mathscr{M}_{1}(I)$ of degree $\left.\frac{1}{2}[2 x(I)+z(I)-v(I)]-\lambda\left|\mathscr{L}_{0}(I)\right|\right]$, which is $\geqq 0$ by $(3.7)$. 
(3) If $\sigma(U(I))=-1$, then (3.33) multiplied with any $H^{\mathrm{I}}(\alpha, \tau)$ is tempered and continuous in (3.1) for $r \downarrow 0$. Here $H^{\mathrm{I}}(\alpha, \tau)$ is homogeneous of degree $\frac{1}{2}[\nu(I)-2 x(I)-z(I)]+\lambda\left|\mathscr{L}_{0}(I)\right|(\geqq 0$ by $(3.8))$ in the variables $A_{i j}^{\tilde{\mathrm{I}}}, U(\tilde{I}) \in \mathfrak{U}_{1}(I) \cup \mathfrak{H}_{1}\left(I^{\prime}\right) \ldots \cup \mathfrak{H}_{1}\left(I^{k}\right)$, and $\alpha_{l}, \quad l \in \mathscr{M}_{1}(I) \cup \cdots$ $\cdots \cup \mathscr{M}_{1}\left(I^{k}\right)$, where $U\left(I^{k}\right)$ is the bough of maximal order containing $U(I)$ and $U\left(I^{k}\right) \supset \cdots \supset U\left(I^{\prime}\right) \supset U(I)$ the chain of twigs between $U(I)$ and $U\left(I^{k}\right)$. These assumptions are satisfied for $s=0$. Consider a branch $U(I)$ with $s(I)=s>0$ and write (3.33) in the form

$$
\prod_{l \in \mathscr{L}(I)-\mathscr{M}} D_{l}^{-(1+\lambda)} \prod_{l \in \mathscr{M}(I)} \alpha_{l}^{(1-\lambda)} R^{I}\left(A^{I}\right) S^{I}\left(B^{I}\right) \prod_{(I, i) \in \mathscr{I}} T^{(I, i)}(\alpha, \tau)
$$

By induction assumption each $T^{(I, i)}(\alpha, \tau)$ is of the form $F^{(I, i)}(\alpha, \tau) \times$ $\times G^{(I, i)}(\alpha, \tau)$, if $\sigma(U(I))=+1$, with $\operatorname{deg} F^{(I, i)}=\frac{1}{2}[2 x(I, i)+z(I, i)-$ $-\nu(I, i)]-\lambda\left|\mathscr{L}_{0}(I, i)\right|$.

Let $U(I)$ be a bough and assume that

is of the form

$$
\prod_{\mathscr{L}(I)-\mathscr{M}} D_{l}^{-1-\lambda} \prod_{\mathscr{M}(I)} \alpha_{l}^{(1-\lambda)} R^{I}\left(A^{I}\right) S^{I}\left(B^{I}\right) \prod_{\sigma(U(I, i)=1} F^{(I, i)}(\alpha, \tau)
$$

$$
F^{I}(\alpha, \tau) K^{I}(\alpha, \tau) \prod_{\sigma(U(I, i))=-1} H^{(I, i)}(\alpha, \tau)
$$

with $F^{I}, H^{(I, i)}$ as in (2), (3), and $K^{I}$ continuous and tempered in (3.1) for $r \downarrow 0$. This is sufficient for performing the induction step, since for all twigs $U(I, i)$ the $H^{(I, i)}$ can be used by $(3)$ to make bounded the $T^{(I, i)}$ (the bough of maximal order containing $U(I, i)$ is $U(I))$. Now

$$
\prod_{\mathscr{M}(I)} \alpha_{l}^{(1-\lambda)} R^{I}\left(A^{I}\right) \prod_{\sigma(U(I, i))=1} F^{(I, i)}(\alpha, \tau)
$$

is homogeneous in $A_{i j}^{I^{\prime}}, U\left(I^{\prime}\right) \in \mathfrak{U}_{1}(I)$, and $\alpha_{l}, l \in \mathscr{M}_{1}(I)$, since for $\sigma(U(I))=+1 \mathfrak{H}_{1}(I, i) \subset \mathfrak{H}_{1}(I)$. The degree of $(3.38)$ is

$$
\begin{gathered}
(1-\lambda)|\mathscr{M}(I)|+x(I)+ \\
+\sum_{\sigma(U(I, i))=1}\left[x(I, i)+\frac{z(I, i)-v(I, i)}{2}-\lambda\left|\mathscr{L}_{0}(I, i)\right|\right] .
\end{gathered}
$$

By (3.7) and (3.8)

$$
\begin{array}{r}
x(I) \geqq \frac{v(I)-z(I)}{2}+\frac{1}{2} \geqq \frac{v(I)-z(I)}{2}+\lambda\left|\mathscr{L}_{0}(I)\right|, \\
z(I) \leqq \sum_{\mathscr{L}(I)} r_{l}+\sum_{(I, i) \in \mathscr{I}}[2 x(I, i)+z(I, i)]-2 y(I),
\end{array}
$$

and (3.39) is larger than

$$
\begin{aligned}
& \geqq(1-\lambda)|\mathscr{M}(I)|+\frac{v(I)}{2}+\lambda\left|\mathscr{L}_{0}(I)\right|-\frac{1}{2} \sum_{\mathscr{L}(I)} r_{l}+y(I)+ \\
& +\sum_{\sigma(U(I, i))=1}\left[x(I, i)+\frac{z(I, i)-v(I, i)}{2}-\lambda\left|\mathscr{L}_{0}(I, i)\right|\right]- \\
& -\sum_{(I, i) \in \mathscr{I}}\left[x(I, i)+\frac{z(I, i)}{2}\right]+\left[x(I)+\frac{z(I)-v(I)}{2}-\lambda\left|\mathscr{L}_{0}(I)\right|\right] .
\end{aligned}
$$


We remark that $\left|\mathscr{V}_{0}(I)\right|-|\mathscr{M}(I)|-1=\sum\left[\left|\mathscr{V}_{0}(I, i)\right|-1\right]$. Therefore we obtain from (3.3)

$$
v(I)=\sum_{(I, i) \in \mathscr{I}} \nu(I, i)+\sum_{\mathscr{L}(I)}\left(r_{l}+2\right)-4|\mathscr{M}(I)| .
$$

Using (3.43) and the relation $\left|\mathscr{L}_{0}(I)\right|-|\mathscr{L}(I)|=\sum\left|\mathscr{L}_{0}(I, i)\right|$ one transforms (3.42) into

$$
\begin{aligned}
= & y(I)+(1+\lambda)[|\mathscr{L}(I)|-|\mathscr{M}(I)|]+x(I)+\frac{z(I)-v(I)}{2}- \\
& -\lambda\left|\mathscr{L}_{0}(I)\right|+\sum_{\sigma(I, i)=-1}\left[\frac{v(I, i)-z(I, i)}{2}-x(I, i)+\lambda\left|\mathscr{L}_{0}(I, i)\right|\right] .
\end{aligned}
$$

Therefore we can split off from (3.38) a factor $F^{I} \cdot \prod_{\sigma(U(I, i))=-1} H^{(I, i)}$. The remainder $L^{I}(\alpha, \tau)$ is homogeneous of degree $\geqq y(I)+(1+\lambda)[|\mathscr{L}(I)|-$ - $|\mathscr{M}(I)|]$ in $\mathrm{A}_{i j}^{r^{\prime}}, U\left(I^{\prime}\right) \in \mathcal{U}_{1}(I)$ and $\alpha_{l}, l \in \mathscr{M}_{1}(I)$. By Lemma 3.1 these $A_{i j}^{I^{\prime}}$ are of the order of the $\alpha_{l}, l \in \mathscr{M}_{1}(I)$. Therefore by Lemma $2.4 L^{I}$ can be used to make bounded the divergent part of (3.36)

$$
\prod_{\mathscr{L}(I)-\mathscr{M}} D_{l}^{-1-\lambda} S^{I}\left(B^{I}\right)
$$

of degree $y(I)+(1+\lambda)[|\mathscr{L}(I)|-|\mathscr{M}(I)|]$.

Finally let $\sigma(U(I))=-1$. Then it follows from the induction hypothesis that

$$
\prod_{\mathscr{M}(I)} \alpha_{l}^{1-\lambda} R^{I}\left(A^{I}\right) \prod_{\sigma(U(I, i))=1} F^{(I, i)}(\alpha, \tau) H^{I}(\alpha, \tau)
$$

is homogeneous in $\alpha_{l}, l \in \mathscr{M}\left(I^{\prime}\right)$, and $A_{i j}^{I^{\prime}}$, where $U\left(I^{\prime}\right) \in \mathfrak{U}_{1}(I) \cup \cdots$ $\cdots \cup \mathfrak{U}_{1}\left(I^{k}\right)$. By Lemma 2.4 and 3.1 one can use (3.46) to make bounded the divergent term $\Pi D_{l}^{-1-\lambda} S^{\mathrm{I}}\left(B^{\mathrm{I}}\right)$ and to produce a factor

$$
\prod_{\sigma(U(I, i))=-1} H^{(I, i)}(\alpha, \tau)
$$

which depends on the right $A_{i j}^{\mathrm{I}^{\prime}}, \alpha_{l}$ for the induction step. A power counting as before shows that the degree of (3.46) is high enough for these operations. Q.E.D.

We remark that in every bough the coefficient of $\exp [\ldots]$ in (3.4) is homogeneous of degree $>-\left|\mathscr{L}_{0}(I)\right|+1$ in $\alpha$.

\section{Renormalization}

We have now accumulated enough information to discuss the limits $\varepsilon \downarrow 0$ and $r \downarrow 0$ in the $\mathscr{R}$-operation and to turn to the properties of the sum of all graph contributions in the perturbation expansion of $\left\langle T \varphi_{1}\left(x_{1}\right) \ldots \varphi_{m}\left(x_{m}\right)\right\rangle^{T}$. 
Let us assume that $\lim _{\varepsilon \downarrow 0} \lim _{r \downarrow 0} \mathscr{R}_{\mathscr{L}}^{r, \varepsilon}\left(V_{1}, \ldots V_{n}\right)$ exists in the topology of $\mathscr{S}^{\prime}\left(R^{4 n}\right)$. For $r, \varepsilon>0$ the subtractions in $\mathscr{R}_{\mathscr{L}}^{r, \varepsilon}\left(V_{1}, \ldots V_{n}\right)$ corresponding to partitions of $\left\{V_{1}, \ldots V_{n}\right\}$ different from $\left\{V_{1}\right\}, \ldots\left\{V_{n}\right\}$ lead to functionals

$$
\varphi \in \mathscr{S}\left(R^{4 n}\right) \rightarrow\left\langle\prod_{j=1}^{k(P)} \mathscr{X}_{\mathscr{L}}^{r, \varepsilon}\left(V_{j 1}^{P}, \ldots V_{j r(j)}^{P}\right) \underset{\text { conn }}{\prod} \Delta_{l}^{r, \varepsilon}, \varphi\right\rangle,
$$

which vanish identically on a certain subspace $\mathscr{S}_{N}\left(R^{4 n}\right) \subset \mathscr{S}\left(R^{4 n}\right)$. Here $N \geqq 0$ is determined by the $\left\{v\left(V_{1}^{\prime}, \ldots V_{m}^{\prime}\right)\right\} . \mathscr{S}_{N}\left(R^{4 n}\right)$ consists of all $\varphi \in \mathscr{S}\left(R^{4 n}\right)$, for which $D \varphi\left(x_{1}, \ldots x_{n}\right)=0$ whenever some $x_{i}=x_{j}$, $1 \leqq i<j \leqq n$, for all differential monomials $D$ in the $\partial / \partial x_{i}^{\mu}$ of degree $\leqq N . \mathscr{S}_{N}\left(R^{4 n}\right)$ is a closed subspace of $\mathscr{S}\left(R^{4 n}\right)$ with the induced topology. It follows from (4.1) that

$$
\lim _{\varepsilon \downarrow 0} \lim _{r \downarrow 0} \mathscr{R}_{\mathscr{L}}^{r, \varepsilon}\left(V_{1}, \ldots V_{n}\right)=\lim _{\varepsilon \downarrow 0} \lim _{r \downarrow 0} \Pi \Delta_{\mathscr{L}}^{r, \varepsilon}
$$

holds on $\mathscr{S}_{N}\left(R^{4 n}\right)$. Therefore [6] $\prod_{\mathscr{L}} \Delta_{l}^{F}$ is a continuous linear functional on $\mathscr{S}_{N}\left(R^{4 n}\right)$ and $\lim _{\varepsilon \downarrow 0} \lim _{r \downarrow 0} \mathscr{R}_{\mathscr{L}}^{\varepsilon, r}\left(V_{1}, \ldots V_{n}\right)$ is its continuation to $\mathscr{S}\left(R^{4 n}\right)$.

By Lemma $3.1 \mathscr{R}_{\mathscr{L}}^{r, \varepsilon}\left(V_{1}, \ldots V_{n}\right)$ is in $p$-space a sum of terms

$$
\delta\left(\sum_{i=1}^{n} p_{i}\right) P(p) Q(\alpha, \tau) \exp \left[i \sum_{i j=1}^{n-1} A_{i j} p_{i} p_{j}-i \sum_{\mathscr{L}} \alpha_{l}\left(m_{l}^{2}-i \varepsilon\right)\right]
$$

integrated over all $0 \leqq \tau(I) \leqq 1$ and over a sector (3.1). By Lemma 3.2 the $A_{i j}=A_{i j}(\alpha, \tau)$ are continuous in (3.1) for $r \downarrow 0$ and homogeneous of degree +1 in the $\alpha . P(p)$ is a monomial in $p_{1}, \ldots p_{n-1} ; Q(\alpha, \tau)$ is rational, homogeneous of degree $d \geqq(-L+1)$ in $\alpha$, tempered and locally integrable in (3.1) for $r \downarrow 0$. Therefore $\lim _{r \downarrow 0} \mathscr{R}_{\mathscr{L}}^{r, \varepsilon}\left(V_{1}, \ldots V_{n}\right)$ exists in the ordinary sense and is (apart from $\delta\left(\sum^{\prime} p_{i}\right)$ ) a function in $\mathcal{O}_{M}\left(R^{4(n-1)}\right)$.

We could have chosen a more general regularization for $\widetilde{\Delta}_{l}^{F}(p)$

$$
\widetilde{\Delta}_{l}^{\rho, \varepsilon}(p)=P_{l}(p) \int_{0}^{\infty} d \alpha f_{\varrho}(\alpha) \exp \left[i \alpha\left(p^{2}-m_{l}^{2}+i \varepsilon\right)\right],
$$

where $f_{\varrho}(\alpha)$ is continuous and vanishes of sufficiently high order for $\alpha \downarrow 0$ for all $\varrho$ in, say, $0<\varrho \leqq 1$, where $\left|f_{\varrho}(\alpha)\right|<c(1+|\alpha|)^{N}$ for some $c, N<\infty$ uniformly for all $0 \leqq \varrho \leqq 1$ and $0 \leqq \alpha<\infty$ and where $f_{\varrho}(\alpha) \rightarrow 1$ pointwise for $\varrho \downarrow 0$. Then one obtains for an obviously defined $\tilde{\mathscr{R}}^{\varrho}, \varepsilon_{\text {-operation }}$

$$
\lim _{\varrho \downarrow 0} \tilde{\mathscr{R}}_{\mathscr{\mathscr { L }}}^{\rho, \varepsilon}\left(V_{1}, \ldots V_{n}\right)=\lim _{r \downarrow 0} \mathscr{R}_{\mathscr{L}}^{r, \varepsilon}\left(V_{1}, \ldots V_{n}\right)
$$

by the Lebesgue dominated convergence theorem. 
The limit $\varepsilon \downarrow 0$ of $\mathscr{R}_{\mathscr{L}}^{0, \varepsilon}\left(V_{1}, \ldots V_{n}\right)$ in $\mathscr{S}^{\prime}\left(R^{4 n}\right)$ has been discussed in [1], [2]. Unfortunately the argument relies on a splitting of the testing functions $\varphi \in \mathscr{S}\left(R^{4 n}\right)$, (see [1], (4.39)), which is in general impossible. But the limit can be expressed directly in terms of elementary distributions. For the proof we discuss a more general situation: We restrict in (4.3) the $\left(p_{1}, \ldots p_{n-1}\right)$ to some $m$-dimensional linear manifold, $0 \leqq m \leqq 4(n-1)$, by setting

$$
p_{i}^{\mu}=\sum_{j=1}^{m} c_{j, 4(i-1)+\mu} q_{j}, 1 \leqq i \leqq n-1,0 \leqq \mu \leqq 3,
$$

where $\operatorname{rank}\left(c_{j k}\right)=m$. For any $\psi \in \mathscr{S}\left(R^{m}\right)$ we study

$$
\begin{aligned}
F_{\varepsilon}(\psi)=\int & d q_{1} \ldots d q_{m} \psi\left(q_{1}, \ldots q_{m}\right) \int_{0}^{1} \ldots \int_{0}^{1} \Pi d \tau(I) \int_{\alpha_{l_{1}} \geqq \alpha_{l L} \geqq 0} \ldots \iint_{l} \Pi d \alpha_{l} \times \\
& \times P^{\prime}(q) Q(\alpha, \tau) \exp \left[i \sum_{i, j=1}^{m} A_{i j}^{\prime} q_{i} q_{j}-i \sum_{l=1}^{L} \alpha_{l}\left(m_{l}^{2}-i \varepsilon\right)\right] .
\end{aligned}
$$

Here $P^{\prime}(q)=P(p(q))$ and $\sum A_{i j}^{\prime} q_{i} q_{j}=\sum A_{i j} p(q)_{i} p(q)_{j}$. If $\lim _{\varepsilon \downarrow 0} F_{\varepsilon}(\psi)$ exists for all $\psi \in \mathscr{S}\left(R^{m}\right)$, then [3] the mapping $\psi \rightarrow \lim _{\varepsilon \downarrow 0} F_{\varepsilon}(\psi)$ is a distribution in $\mathscr{S}^{\prime}\left(R^{m}\right)$ and is the strong limit for $\varepsilon \downarrow 0$ of $\mathscr{R}_{\mathscr{L}}^{0, \varepsilon}\left(V_{1}, \ldots V_{n}\right)$ with the coefficient of $\delta\left(\sum p_{i}\right)$ restricted in $p$-space to the manifold (4.6).

For $\varepsilon>0$ we can freely interchange integrations in (4.7). After the coordinate transform

$$
\alpha_{l}=\lambda \beta_{l}, 1 \leqq l \leqq L, \sum_{l=1}^{L} \beta_{l}=1, \frac{\partial\left(\alpha_{1}, \ldots \alpha_{L}\right)}{\partial\left(\beta_{1}, \ldots \beta_{L-1}, \lambda\right)}=\lambda^{L-1}
$$

we first integrate (4.7) over $0 \leqq \lambda<\infty$. This leads to the Feynman integral.

$$
\begin{array}{r}
F_{\varepsilon}(\psi)=\int_{0}^{1} \ldots \int_{0}^{1} \Pi d \tau(I) \int_{1 \geqq \beta_{l_{1}} \geqq \cdots \beta_{l L} \geqq 0} \Pi d \beta_{l} \delta\left(1-\sum \beta_{l}\right) Q(\beta, \tau) \times \\
\times F_{\varepsilon}(\psi, \beta, \tau) \\
F_{\varepsilon}(\psi, \beta, \tau)=c \int d q_{1} \ldots d q_{m} \frac{P^{\prime}(q) \psi\left(q_{1}, \ldots q_{m}\right)}{\left[\Sigma A_{i j}^{\prime}(\beta, \tau) q_{i} q_{j}-\Sigma \beta_{l} m^{2}+i \varepsilon\right]^{d+L}} .
\end{array}
$$

Since $Q(\beta, \tau)$ is locally integrable, it is sufficient to show that $\lim _{\varepsilon \downarrow 0} F_{\varepsilon}(\psi, \beta, \tau)$ exists and is continuous in $\beta$ and $\tau$ in the compact region of integration in (4.9). Since $m_{l}^{2}>0,1 \leqq l \leqq L$, the singular support of (4.10)

$$
\left\{\sum_{i, j=1}^{m} A_{i j}^{\prime}(\beta, \tau) q_{i} q_{j}=\sum_{l=1}^{L} \beta_{l} m_{l}^{2}\right\}
$$

is an analytic manifold in $\left(q_{1}, \ldots q_{m}\right)$ for fixed $\beta$ and $\tau$. Thus $\lim _{\varepsilon \downarrow 0} F_{\varepsilon}(\psi, \beta, \tau)$ exists and is in local coordinates expressible in terms of 
derivatives of $\delta$-functions and principal values. As the $A_{i j}^{\prime}(\beta, \tau)$ are continuous in the region of integration in (4.9), the intersection of (4.11) with any compact subset of $R^{m}$ varies continuously with $\beta$ and $\tau$. This proves the continuity of $\lim _{\varepsilon \downarrow 0} F_{\varepsilon}(\psi, \beta, \tau)$ in $\beta$ and $\tau$, since $\psi$ is strongly decreasing at infinity, where (4.11) is well-behaved.

We now return to the Gell-Mann Low expansion (1.1) of $\left\langle T \varphi_{1}\left(x_{1}\right) \ldots\right.$ $\left.\ldots \varphi_{m}\left(x_{m}\right)\right\rangle^{T}$, where we define $\left\langle T \varphi_{1}^{\mathrm{I}}\left(x_{1}\right) \ldots \mathscr{H}^{\mathrm{I}}\left(x_{n}\right)\right\rangle^{T}$ by a sum of $\lim _{\varepsilon \downarrow 0} \lim _{r \downarrow 0} \mathscr{R}_{\mathscr{L}}^{r \varepsilon}\left(V_{1}, \ldots V_{n}\right)$. The integration over $x_{m+1}, \ldots x_{n}$ in (1.1) corresponds in $p$-space to a restriction to a $4 m$-dimensional linear manifold in $R^{4 n}$. The preceeding argument applies:

Theorem: The pertubation-theoretic Green's functions $\left\langle T \varphi_{1}\left(x_{1}\right) \ldots\right.$ $\left.\ldots \varphi_{m}\left(x_{m}\right)\right\rangle_{(n)}^{T}$ of order $n$ defined by the $\mathscr{R}$-operation are in $p$-space (up to $\delta\left(\sum p_{i}\right)$ ) Lorentz covariant boundary values in $\mathscr{S}^{\prime}\left(R^{4(m-1)}\right)$ of sums of Feynman integrals, which are analytic in $p_{1}, \ldots p_{m-1}$ without natural boundaries.

Remark: The analyticity properties in $p$-space follow from (4.3). Landau rules for these renormalized Feynman amplitudes can be worked out, but will not be discussed here.

The combinatorial structure of the subtractions in the $\mathscr{R}$-operation and their relation to formal counter terms in $\mathscr{H}^{\mathrm{I}}(x)$ have been lucidly treated by Bogoliubov and SHIRkov [10]. It is noteworthy that the $\mathscr{R}$-operation can also be applied to theories, which are conventionally considered as non-renormalizable. The distinction of the renormalizable theories in the restricted sense follows from the usual power counting theorem [7], [10], by which these theories have counter terms to $\mathscr{H}^{\mathrm{I}}(x)$, which involve only a finite number of WICK polynomials in the $\varphi_{i}^{\mathrm{I}}(x)$ uniformly for all orders $n$.

Example: As an illustration of the method of Bogoliubov and PARASIUK we shall discuss the quartic self-interaction of a neutral scalar field. It is generally believed that the $\varphi^{4}$-theory [9] is in perturbation theory up to order $n$ uniquely characterized by renormalizing

$$
\tau\left(x_{1}, \ldots x_{m}\right)_{(n)}^{T}=\sum_{k=0}^{n} \frac{(-i)^{k}}{k !} \int d y_{1} \ldots d y_{k}\left\langle T \varphi^{\mathrm{I}}\left(x_{1}\right) \ldots \mathscr{H}^{\mathrm{I}}\left(y_{k}\right)\right\rangle^{T}
$$

using counter terms to $\mathscr{H}^{\mathrm{I}}(y)=-\frac{g}{4 !}: \varphi^{\mathrm{I}}(y)^{4}:$ of the type

$$
A: \varphi^{\mathrm{I}}(y)^{2}:+B \square: \varphi^{\mathrm{I}}(y)^{2}:+C: \varphi^{\mathrm{I}}(y)^{4}: .
$$

Here $g$ is the physical coupling constant and $\varphi^{\mathrm{I}}(y)$ is a neutral scalar 
free field with the physical mass $m$ and

$$
\begin{aligned}
\left\langle T \varphi^{\mathrm{I}}\left(x_{1}\right) \varphi^{\mathrm{I}}\left(x_{2}\right)\right\rangle^{T} & =i \Delta^{F}\left(m ; x_{1}-x_{2}\right) \\
& =\frac{i}{(2 \pi)^{4}} \int \frac{d p \exp \left[-i p\left(x_{1}-x_{2}\right)\right]}{p^{2}-m^{2}+i 0} .
\end{aligned}
$$

The "renormalization constants"

$$
A=\sum_{k=2}^{n} A_{k} g^{k}, B=\sum_{k=2}^{n} B_{k} g^{k}, C=\sum_{k=2}^{n} C_{k} g^{k}
$$

are determined by three conditions on the Green's functions $\tau\left(x_{1}, \ldots x_{m}\right)_{(k)}^{T}$ for $2 \leqq k \leqq n:(A, B):$ The 2 -point function $\tilde{\tau}\left(p_{1}, p_{2}\right)_{(k)}^{T}=\delta\left(p_{1}+p_{2}\right) \tilde{\tau}\left(p_{1}^{2}\right)_{(k)}$ must have a pole at $p_{1}^{2}=m^{2}$ with residue $(2 \pi)^{-2}$

$$
(2 \pi)^{2}\left(p_{1}^{2}-m^{2}\right) \tilde{\tau}\left(p_{1}^{2}\right)_{(k)}=1 \quad \text { for } \quad p_{1}^{2}=m^{2} .
$$

$(C)$ : The 2-body scattering amplitude

$$
\begin{aligned}
& \left\langle p_{1}, p_{2}^{\text {out }} \mid-p_{3},-p_{4}^{\text {in }}\right\rangle_{(k)}-\left\langle p_{1}, p_{2}^{\text {in }} \mid-p_{3},-p_{4}^{\text {in }}\right\rangle_{(k)} \\
& \quad=i \delta\left(\sum p_{i}\right) \tilde{T}\left(p_{1}, \ldots p_{4}\right)_{(k)}=(2 \pi)^{2} \prod_{i=1}^{4}\left(p_{i}^{2}-m^{2}\right) \tilde{\tau}\left(p_{1}, \ldots p_{4}\right)_{(k)}^{T}
\end{aligned}
$$

is normalized at $p_{i}^{2}=m^{2},\left(p_{i}+p_{j}\right)^{2}=\frac{4 m^{2}}{3}, i<j$, to

$$
\tilde{T}\left(p_{1}, \ldots p_{4}\right)_{(k)}=\frac{g}{(2 \pi)^{2}} .
$$

The conditions (4.14), (4.16) are satisfied in order $n<2$, but not for general $n$, if we define $\tau\left(x_{1}, \ldots x_{m}\right)_{(n)}^{T}$ by the $\mathscr{R}$-operation of section 1 . The remedy is to use the freedom of the choice of the point around which the Taylor expansion is taken in (1.9). The results of the preceeding sections remain valid, if one defines a more general $\mathscr{R}$-operation [10] by adding recursively to any $-M \overline{\mathscr{R}}_{\mathscr{L}}^{r, \varepsilon}\left(V_{1}^{\prime}, \ldots V_{m}^{\prime}\right)$ a distribution $\tilde{\mathscr{X}}_{\mathscr{L}}\left(V_{1}^{\prime} \ldots V_{m}^{\prime}\right)$, which is in $p$-space of the form $\delta\left(\sum p_{i}^{\prime}\right) \widetilde{P}\left(p_{1}^{\prime}, \ldots p_{m}^{\prime}\right)$ with a covariant polynomial $\widetilde{P}$ of degree $\leqq v\left(V_{1}^{\prime}, \ldots V_{m}^{\prime}\right)$ depending only on the structure of $G\left(V_{1}^{\prime}, \ldots V_{m}^{\prime}, \mathscr{L}\right)$.

Theorem: In the $\varphi^{4}$-theory there exists a choice of the finite renormalizations consistent with (4.14) and (4.16), and any such choice leads to the same renormalized Green's distributions $\tau\left(x_{1}, \ldots x_{m}\right)_{(n)}^{T}$ for all $m, n$.

Proof: In the $\varphi^{4}$-theory $v\left(V_{1}^{\prime}, \ldots V_{m}^{\prime}\right)=2$ or 0 for subgraphs $G\left(V_{1}^{\prime}, \ldots V_{m}^{\prime}, \mathscr{L}\right)$ with two or four external lines, respectively. Otherwise no over-all subtractions are necessary.

We can always enforce (4.14) and (4.16), since the renormalized Feynman amplitudes for the self energy (SE) and vertex (V) parts are 
analytic around $p_{1}^{2}=m^{2}$ and the Chew-Mandelstam point, respectively. Furthermore in any order $n \geqq 2$ there exist SE- and V-parts, which require a new over-all subtraction, e.g. the graphs of Fig. 7. In second
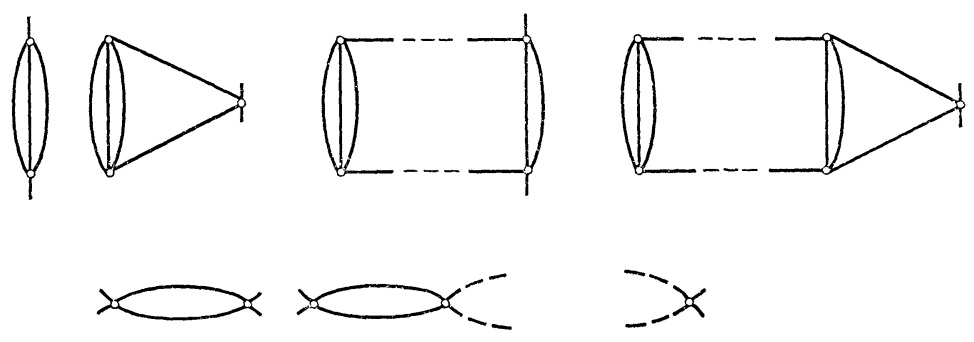

Fig. 7. $S E$ - and $V$-parts

order the finite renormalizations are uniquely determined by (4.14) and (4.16), since there is only one divergent SE- and V-part. In third order the following SE-parts require an over-all subtraction:

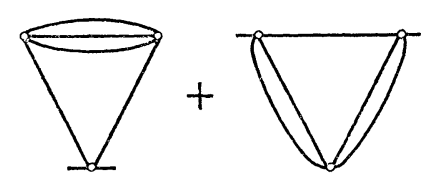

Fig. 8. Independent $S E$-parts in 3rd order

Only the sum of the counter terms, a polynomial $A_{3}^{r, \varepsilon}+B_{3}^{r, \varepsilon} p_{1}^{2}$, is uniquely determined. We shall see that this "renormalization gauge" leaves the renormalized Green's functions (4.12) invariant.

Consider all graphs $\mathfrak{G}_{m, n}$ of $n^{t h}$ order in the Wick expansion of (4.12). The analytical contribution of each graph is defined by the $\mathscr{R}$-operation, where the combinatorics of the counter terms is determined
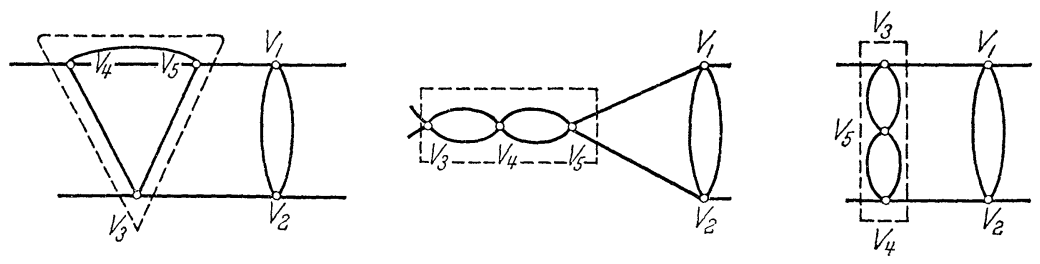

Fig. 9. Typical terms in $\mathfrak{S}_{\gamma}\left(V_{1}, V_{2},\left\{V_{3}, V_{4}, V_{5}\right\}\right)$

by the internal vertices $V_{1}, \ldots V_{n}$. For any partition

$$
U_{1}=\left\{V_{11}, \ldots V_{1 r(1)}\right\}, \ldots U_{s}=\left\{V_{s 1}, \ldots V_{s r(s)}\right\}
$$

of $\left\{V_{1}, \ldots V_{n}\right\}$ into $s<n$ generalized vertices a certain subclass $\mathfrak{G}\left(U_{1}, \ldots U_{s}\right)$ of $\mathfrak{G}_{m, n}$ requires a subtraction. This set falls into equi- 
valence classes $\mathfrak{G}_{\gamma}\left(U_{1}, \ldots U_{s}\right)$, where two graphs $G_{1}, G_{2} \in \mathfrak{G}_{\gamma}\left(U_{1}, \ldots U_{s}\right)$ differ only in the structure of the internal lines and vertices in $U_{1}, \ldots U_{s}$.

Example: Graphs belonging to the same $\mathfrak{G}_{\gamma}\left(V_{1}, V_{2},\left\{V_{3}, V_{4}, V_{5}\right\}\right)$. The Wick expansion of $\left\langle T \varphi^{I}\left(x_{1}\right) \ldots \mathscr{H}^{I}\left(y_{n}\right)\right\rangle^{T}$ contains all possible connected contractions, where the $m$ lines from $x_{1}, \ldots x_{m}$ and the $4 n$ lines from $y_{1}, \ldots y_{n}$ connect different vertices. Thus $\mathfrak{G}_{\gamma}\left(U_{1}, \ldots U_{s}\right)$ is either empty or contains exactly once all possible contractions between the $4 r(j)$ lines from the $r(j)$ vertices in $U_{j}, 1 \leqq j \leqq s$, which connect different vertices and lead to IPI SE- or V-parts, respectively.

The analytical form of the sums of counter terms for all graphs in $\mathfrak{G}_{\gamma}\left(U_{1}, \ldots U_{s}\right)$, which arise from the subtractions associated with the generalized vertices $U_{1}, \ldots U_{s}$, is for $r, \varepsilon>0$ in $p$-space of the form

$$
\delta\left(\sum p_{i}\right) \int d k_{1} \ldots d k_{t} \prod_{\text {conn }} \widetilde{\Delta}_{l}^{r, \varepsilon}\left(q^{\prime}\right) \prod_{U_{i}: S E}\left(A_{r^{\prime}(i)}^{r, \varepsilon}+B_{r(i)}^{r, \varepsilon} q^{\prime \prime 2}\right) \prod_{U_{j}: V} C_{r(j)}^{r, \varepsilon},
$$

where $k_{1}, \ldots k_{t}$ are loop momenta, the $q^{\prime}, q^{\prime \prime}$ are linear combinations of the $k_{1}, \ldots k_{t}$ and the external momenta $p_{1}, \ldots p_{m}, \prod_{\text {conn }}$ extends over all lines which are not contained in $U_{1}, \ldots U_{s}, \prod_{U_{i}: S E}$ over all SE-parts $U_{i}$ and $\prod_{U_{j}: V}$ over all V-parts $U_{j}$ (with $r(j)>1$ ) of the partition $U_{1}, \ldots U_{s}$. The essential observation is that the SE-contributions $A_{r(i)}^{r, \varepsilon}+B_{r(i)}^{r, \varepsilon} q^{\prime \prime 2}$ in (4.17) are exactly the same as the sum of all over-all subtractions in $\mathscr{R}_{\mathscr{E}}^{r_{\mathscr{E}} \varepsilon} \tau\left(x_{1}, x_{2}\right)_{(r(i))}^{T}$ from order $r(i)$ and similarly for $C_{r(i)}^{r, \varepsilon}$ in any V-part $U_{j}$.

We know that $A_{n}^{r, \varepsilon}, B_{n}^{r, \varepsilon}, C_{n}^{r, \varepsilon}$ are uniquely determined by (4.14), (4.16) for $n=2$. Assume that the same holds in orders $2 \leqq k<n, n \geqq 3$, and consider all graphs contributing to $\left\langle T \varphi^{I}\left(x_{1}\right) \varphi^{I}\left(x_{2}\right) \mathscr{H}^{I}\left(y_{1}\right) \ldots\right.$ $\left.\ldots \mathscr{H}^{I}\left(y_{n}\right)\right\rangle^{T}$. The sums of subtractions associated with all partitions $U_{1}, \ldots U_{s}$ of $\left\{V_{1}, \ldots V_{n}\right\}, s>1$, are uniquely determined by (4.17) using the induction assumption. The sum of the over-all subtractions for $\mathfrak{G}\left(\left\{V_{1}, \ldots V_{n}\right\}\right)$ of the form $\delta\left(p_{1}+p_{2}\right) \Delta^{r, \varepsilon}\left(p_{1}\right)^{2}\left[A_{n}^{r, \varepsilon}+B_{n}^{r, \varepsilon} p_{1}^{2}\right]$ is then uniquely determined by (4.14), and similarly $C_{n}^{r, \varepsilon}$ by (4.16). Furthermore this construction is independent of the regularization (4.4).

This proves in the $\varphi^{4}$-theory that the "universal" subtraction procedure of Bogoliubov and Parasiuk together with the adjustment of three parameters in any order $n$ leads to unique time-ordered distributions.

The author is greatly indebted to Professor A. S. Wightman, whose penetrating lectures on renormalization theory were a continuous challenge for his students. Without his encouragement and criticism this paper could not possibly be written. For many clarifying discussions I am grateful to Professors and Doctors J. Challifour, F. J. Dyson, H. Epstein, A. Jaffe, A. S. Wightman, C. N. Yang and W. Zimmermann. It is a pleasure to thank Professor J. R. Oppenheimer for his kind hospitality at The Institute for Advanced Study. 
326 K. HEPP: Proof of the Bogoliubov-Parasiuk Theorem on Renormalization

\section{References}

[1] Bogoliubov, N. N., and O. S. Parasiuk: Acta Math. 97, 227 (1957).

[2] Parasiuk, O. S.: Ukrainskii Math. J. 12, 287 (1960).

[3] Schwartz, L.: Théorie des distributions, I, II. Paris: Hermann 1957/59.

[4] Wrck, G. C.: Phys. Rev. 80, 268 (1950).

[5] Pauli, W., and F. Villars: Rev. Mod. Phys. 21, 434 (1949).

[6] Parasiuk, O. S.: Izv. Akad. Nauk, Ser. Mat. 20, 843 (1956).

[7] Dyson, F. J.: Phys. Rev. 75, 486 and 1736 (1949).

[8] Salam, A.: Phys. Rev. 82, 217; 84, 426 (1951).

[9] Wu, T. T.: Phys. Rev. 125, 1436 (1962).

[10] Bogoliubov, N. N., and D. V. ShiRkov: Introduction to the theory of quantized fields. New York: Interscience 1959. 